\title{
Explicación y Predicción de la Inflación en Mercados Emergentes: El Caso de México:
}

\author{
Jeannine Bailliu, Daniel Garcés Díaz, Mark Kruger y Miguel Messmacher^ \\ Bank of Canada y Banco de México
}

Febrero de 2003

Documento de Investigación No. 2003-3

Dirección General de Investigación Económica

BANCO DE MÉXICO

\footnotetext{
* Este artículo es un producto del programa de investigación conjunta entre el Banco de México y el Banco de Canadá aunque las opiniones contenidas en él son las de los autores y no necesariamente las de las instituciones que lo patrocinaron. Los autores reconocen la responsabilidad de cualquier error u omisión y agradecen a Brigitte Desroches y Rocío Elizondo por su excelente ayuda en todas las etapas del trabajo, lo mismo que a Armando Baqueiro y a los participantes en los seminarios llevados a cabo en el Banco de México y en el Banco de Canadá por sus comentarios y sugerencias.

- Correspondencia: Jeannine Bailliu, International Department, Bank of Canada, 234 Wellington Street, Ottawa, Ontario, K1A 0G9, Canada. Tel.: (613) 782-7474; Fax: (613) 782-7658; E-mail: jbailliu@ bankofcanada.ca y Daniel Garcés Díaz, Banco de México, Av. 5 de Mayo \# 18, México, D.F., C.P. 06059. Tel. (52) 5237-2577; Fax: (52) 5237-2687; Email dgarces@banxico.org.mx.
} 


\title{
Explicación y Predicción de la Inflación en Mercados Emergentes: El Caso de México
}

\author{
Jeannine Bailliu, Daniel Garcés Díaz, Mark Kruger y Miguel Messmacher \\ Bank of Canada y Banco de México
}

Febrero de 2003

Documento de Investigación No. 2003-3

Dirección General de Investigación Económica

BANCO DE MÉXICO

\begin{abstract}
$\underline{\text { Resumen }}$
En este artículo se evalúa la precisión de los pronósticos generados por tres tipos de modelos para la inflación en México durante el período 1983-2001. Estos modelos son el margen sobre costo marginal (markup), la curva de Phillips clásica aumentada con tipo de cambio y el modelo de la brecha de dinero. Los modelos se comparan entre sí y contra el estándar mínimo del mejor modelo puro de series de tiempo disponible. Los resultados indican que el modelo del margen sobre costo marginal supera ampliamente a los demás mientras que el poder explicativo de la curva de Phillips clásica solamente supera al del modelo puro de series de tiempo si se incluye al tipo de cambio. El modelo de la brecha de dinero no produce buenos resultados, indicando que el paradigma de "dinero pasivo" es aplicable a México. El artículo concluye con una discusión del futuro de estos modelos en un ambiente de inflación baja y estable.
\end{abstract}




\section{Introducción}

En años recientes, muchos países en vías de desarrollo han experimentado reducciones dramáticas de sus niveles de inflación como resultado de factores externos relativamente benignos y de la instrumentación de políticas internas responsables. En algunos países, la desinflación se logró con la adopción de un régimen de tipo de cambio controlado. Mientras que este régimen monetario típicamente se traduce en precios internos más estables, a menudo ha conllevado problemas de balanza de pagos. Como consecuencia de lo anterior, ha surgido una tendencia a abandonar al tipo de cambio como ancla nominal. Algunos países de mercados emergentes (por ejemplo Brasil, Chile, Israel y México) han decidido seguir la pauta de varios países industrializados y adoptar una combinación de políticas de tipo de cambio flotante y objetivos de inflación. Sin embargo, la aplicación exitosa dichas políticas presupone que el banco central tiene un adecuado entendimiento de la dinámica de la inflación interna y puede pronosticar eficazmente su trayectoria futura.

Hay una extensa literatura sobre las causas de la inflación y varios modelos compiten para representar el proceso inflacionario. El propósito de este artículo es aplicar modelos de inflación comúnmente usados en los países industrializados al caso de México, un país en desarrollo que recientemente adoptó el esquema de objetivos de inflación como marco de su política monetaria. Se compara el desempeño de estos modelos con el de un modelo de margen de sobrecosto (markup) que ha sido usado exitosamente para el análisis de la inflación en México. El enfoque en este documento es enfatizar el uso de modelos que tengan sustento teórico y, que puedan ser útiles para entender las causas de la inflación y para producir pronósticos razonables. ${ }^{1}$ Más aún, debido a la incertidumbre inherente en la estimación de cualquier modelo particular, es importante comparar las predicciones de varios modelos. Este es el enfoque preferido en muchos bancos centrales. Por ejemplo, Blinder (1998, p.12) señala que cuando enfrentaba la incertidumbre sobre modelos mientras se encontraba sirviendo en la junta de la Reserva Federal él se inclinaba por: "Usar una amplia variedad de modelos...Mi procedimiento usual fue el de simular una política particular con tantos de estos modelos como fuese posible..." Posteriormente, el juicio personal es utilizado al evaluar los resultados de cada modelo. Similarmente, Longworth y Freedman (2000) advierten que, dada la incertidumbre del modelo en un medio cambiante, es importante para los bancos centrales tener como referencia una variedad de modelos al conducir la política monetaria.

El artículo se organiza de la siguiente manera. En la sección 2 se revisan las causas de la inflación tanto en países industrializados como en países en vías de desarrollo, con énfasis en estudios previos realizados para México; en la sección 3 se presentan los tres tipos de modelos de

\footnotetext{
${ }^{1}$ El enfoque de este trabajo puede considerarse como un camino intermedio entre desarrollar un modelo estructural que se encuentra completamente especificado pero que no es muy útil para pronosticar y uno que enfatiza especificaciones puras de series de tiempo con el único propósito de encontrar el modelo de pronóstico óptimo.
} 
inflación en los que se basa este trabajo. La sección 4 contiene los resultados de la estimación de los modelos, y en la sección 5 se compara su desempeño para pronosticar. En la sección 6 se exploran las implicaciones para México en un ambiente de baja inflación. La sección 7 ofrece comentarios finales y las conclusiones.

\section{Causas de la Inflación}

Existe un consenso de que, en el largo plazo, la inflación es un fenómeno puramente monetario. Sin embargo, en el horizonte relevante para la política monetaria, la inflación puede ser afectada por otras variables. Perturbaciones que aumentan la demanda agregada relativamente a la oferta agregada son inflacionarias; también lo son cambios inesperados en variables internas como los salarios y los precios de los bienes importados. Más aún, movimientos del tipo de cambio inciden en la inflación a través del precio de las importaciones $\mathrm{y}$, posiblemente, afectando las expectativas y la conducta de los agentes que fijan precios. ${ }^{2}$ En esta sección se revisa el trabajo teórico y empírico llevado a cabo para identificar las causas de la inflación más relevantes tanto en países industrializados como en naciones en vías de desarrollo.

La curva de Phillips predice que la inflación se relacionará con perturbaciones de factores reales a las curvas de oferta y demanda agregadas. Efectivamente, al examinarse la experiencia de países industrializados y en desarrollo entre 1960 y 1995, el FMI (1996) halló que la brecha de producto explicaba una porción substancial de los movimientos de mediano y largo plazos de la inflación en los países industrializados. No obstante, la validación monetaria a perturbaciones de oferta adversas (como incrementos en el precio del petróleo) explicaban una parte sustancial del aumento de la inflación en los países industrializados en los setenta, debido a que un alza inicial en el nivel de precios alimentó las expectativas inflacionarias y, con ello, el costo posterior de la desinflación. Este episodio enfatiza porqué es importante para las autoridades monetarias ser capaces de distinguir entre perturbaciones de oferta y de demanda.

Mientras que los factores reales son señalados como los determinantes principales de la inflación en los países industrializados, el énfasis primario de la literatura sobre inflación en países en desarrollo ha sido dirigido a factores nominales. Por ejemplo, el FMI (1996) encontró que la brecha de producto explicaba poco de los movimientos de la inflación en los países en desarrollo. La inflación era explicada más bien por variaciones en la tasa de crecimiento del dinero y en el tipo de cambio nominal. Esto no quiere decir que la inflación no pueda ser afectada por la demanda agregada sino que la contribución de ésta es pequeña con relación a la de las perturbaciones nominales; en particular, la inflación en el mediano plazo es vista como el resultado del

\footnotetext{
${ }^{2}$ El FMI (1996) señala que el tipo de cambio y los precios internacionales de los productos de importación se pueden considerar como perturbaciones reales puesto que incrementan el precio de los insumos importados.
} 
financiamiento del déficit gubernamental a través de la creación de dinero o de una política monetaria incongruente. ${ }^{3}$

Otro aspecto clave en esta literatura es el uso del señoreaje por parte del gobierno para financiar su déficit. ${ }^{4}$ Señoreaje es el ingreso que obtiene el gobierno vía incrementos en los saldos monetarios y la pérdida del poder adquisitivo de las tenencias de efectivo (el "impuesto inflacionario"). Puesto de modo simple, el gobierno imprime dinero para pagar sus cuentas y este aumento de la oferta monetaria se traduce en inflación al no haber una expansión correspondiente de la producción de bienes y servicios.

En muchos países de mercados emergentes, especialmente aquéllos con sistemas de recaudación fiscal poco eficientes, el señoreaje es una fuente de recursos muy atractiva para el gobierno. Es más fácil de recaudar que otros tipos de impuestos y no requiere de la aprobación del cuerpo legislativo para ser implementado. Sin embargo, la habilidad del gobierno para obtener el señoreaje se verá restringida si la "base impositiva" es pequeña, esto es, cuando la tenencia de moneda nacional es baja (por ejemplo en economías dolarizadas). En adición, en aquellos países donde el público tiene poca tolerancia hacia la inflación, el gobierno enfrentará la desaprobación de la gente si financia sus déficit a través del señoreaje.

No obstante las ligas teóricas, la evidencia empírica de la conexión entre los déficit, el señoreaje y la inflación ha sido elusiva. Fischer, Sahay y Vegh (2001) señalan que la relación entre el déficit fiscal y la inflación es fuerte solamente en países de alta inflación (o durante episodios de inflación elevada). Sin embargo, también encuentran que no hay una relación obvia entre los déficit y la inflación durante episodios de baja inflación o para países con baja inflación. La ausencia de una relación estrecha para países de baja inflación podría obedecer a la habilidad de sus gobiernos para conseguir préstamos internos. ${ }^{5,6}$ En este caso, la transferencia de recursos del sector privado al sector público no causaría inflación.

En un trabajo más reciente, sin embargo, Catão y Terrones (2001) relacionan exitosamente la inflación de largo plazo con el componente permanente del déficit fiscal reescalado con la "base impositiva" de la inflación, medida como el cociente de dinero estrecho en términos reales y el PIB.

\footnotetext{
${ }^{3}$ Los episodios de macrodevaluaciones que se han traducido en inflación más alta en los mercados emergentes son con frecuencia un reflejo de estos dos canales. Si el gobierno financia su déficit a través de endeudamiento externo llevando a una ampliación del déficit en la cuenta corriente, el país se vuelve más vulnerable a perturbaciones externas y la probabilidad de una crisis de balanza de pagos aumenta. Una política incongruente en el contexto de una política monetaria llevará a una corrida sobre las reserves internacionales y, eventualmente, a una devaluación.

4 Para una revisión de la literatura, véase Fischer (1994).

5 Los países con una inflación baja típicamente poseen mercados locales de capital más desarrollados. El financiamiento externo del gobierno puede en sí mismo ser inflacionario si las exportaciones netas no son reducidas por una apreciación del tipo de cambio nominal.

${ }^{6}$ Sin embargo, si el déficit fiscal ha sido considerable y persistente, la posibilidad de una crisis de solvencia eventualmente se producirá cuando el sector privado no estuviera dispuesto a continuar otorgando financiamiento.
} 
Ellos encuentran que una reducción de 1 por ciento del cociente del déficit fiscal al PIB típicamente baja la inflación de 1.5 a 6 puntos porcentuales dependiendo del tamaño de la oferta monetaria.

En la literatura también se ha examinado la hipótesis de que la carencia de independencia del banco central puede dar lugar a consideraciones de índole política que podrían causar que la política monetaria fuese más relajada que lo necesario. Por ejemplo, si se cree que el producto puede ser aumentado con una política monetaria expansiva, los políticos podrían presionar al banco central, por ejemplo en tiempos electorales, a canjear un impulso al crecimiento por una mayor inflación. Efectivamente, el FMI (1996) muestra que el desempeño inflacionario entre 1970 y 1995 en los países industriales está negativamente correlacionado con un índice de independencia de bancos centrales. Sin embargo, en dicho estudio se concluye que esa relación no se observa durante el mismo período para países en desarrollo. Esto podría deberse a la imprecisión de la medición de la independencia del banco central, puesto que hay una divergencia entre las definiciones de jure y de facto en varios de estos países. ${ }^{7}$

En un estudio más reciente, sin embargo, Jácome (2001) obtiene una correlación negativa entre una autonomía aumentada del banco central y la inflación durante 1999-2001 en América Latina. Ese autor construyó un índice de independencia del banco central para 14 países latinoamericanos que amplió su trabajo previo al incluir criterios de responsabilidad de prestamista de última instancia en adición a otras medidas de independencia de jure. En este índice, una política discrecional de préstamos de emergencia podría perjudicar el logro del control de la inflación, mientras que un papel más limitado de los préstamos del banco central a los bancos comerciales lo reforzaría. ${ }^{8}$

Los movimientos del tipo de cambio pueden afectar a la inflación directamente al elevar los precios de las importaciones e indirectamente al cambiar las expectativas inflacionarias. Así, el pass-through de una depreciación a los precios internos podría ser mucho mayor que lo que la proporción de bienes importados en la canasta de bienes de consumo podría sugerir. Más aún, las expectativas de inflación también pueden afectar el tipo de cambio. Un deterioro de las expectativas inflacionarias tendería a depreciar el tipo de cambio al motivar a los agentes a adquirir moneda extranjera en un esfuerzo para preservar su propio poder adquisitivo. Debido a la retroalimentación entre el tipo de cambio y los precios internos, un país puede caer en un círculo vicioso de depreciación e inflación. En tales situaciones, muchos países han optado por fijar sus tipos de cambio en un esfuerzo para romper este ciclo. Mientras que esta estrategia es a menudo exitosa en el corto plazo, más adelante puede llevar a problemas de balanza de pagos si las políticas macroeconómicas no son compatibles con el régimen de tipo de cambio.

\footnotetext{
${ }^{7}$ La independencia es típicamente medida evaluando la legislación del banco central y su estructura institucional.

${ }^{8}$ Argentina, Perú y Uruguay fueron los casos más notables en este aspecto.
} 
La evolución de las expectativas constituye una parte importante del proceso de la inflación. Una inflación elevada puede llevar a cambios institucionales que incrementan la persistencia inflacionaria. Por ejemplo, la indexación de precios y salarios a aumentos de precios en el pasado incrementa la inercia de la inflación. En adición, si las tasas de interés nominal de la deuda pública son altas debido a que reflejan pronósticos de inflación futura al alza, el gobierno tiene un incentivo para validarlas para mantener el valor real de la deuda. ${ }^{9}$

Algunos estudios empíricos han tratado de identificar las fuentes de inflación más importantes en los mercados emergentes. Lougani and Swagel (2001) examinan la experiencia de 53 países en desarrollo entre 1964 y 1998. Ellos estiman vectores autorregresivos (VARs) con seis variables que incluyen el precio del petróleo, los precios de las mercancías no petroleras, un estimado de la brecha de producto, el crecimiento del dinero, la depreciación del tipo de cambio y la inflación. Encuentran que el crecimiento monetario o bien los movimientos del tipo de cambio (dependiendo del orden) dan cuenta de dos tercios de la varianza de la inflación en horizontes cortos y largos. Los autores muestran que las expectativas también juegan un papel importante en la determinación de la inflación pues el desempeño previo de la misma explica de entre 10 y $20 \%$ de sus movimientos. ${ }^{10}$ En el agregado, las perturbaciones de costos o de la brecha de producto no son factores significativos. Al desagregar su muestra, los autores obtienen que en países con regímenes de tipo de cambio fijo la inflación tiende a tener un componente inercial substancial, mientras que el dinero y el tipo de cambio juegan un papel más relevante en países que flotan.

En contraste, a partir de un examen de la experiencia de 14 países emergentes en los ochenta y noventa, Mohanty y Klau (2001) encuentran que perturbaciones exógenas de oferta, en particular de los precios de los alimentos, son un determinante de la inflación. Los precios de los alimentos típicamente componen un porcentaje mayor del índice de precios al consumidor en un país de mercado emergente que en un país desarrollado. Más aún, tales precios son altamente volátiles debido a la influencia del clima y a la presencia de restricciones comerciales. Los factores de demanda, representados por la brecha de producto y el desequilibrio monetario, resultaron significativos. Sin embargo, la inflación salarial y las variaciones del tipo de cambio fueron vistas como factores importantes de la volatilidad inflacionaria en muchos países. Los autores también encuentran que la persistencia inflacionaria juega un papel mayor en explicar tanto el nivel promedio como la variación de la inflación.

\footnotetext{
9 Una reducción en la inflación incrementaría el valor real de la deuda.

${ }^{10}$ Una explicación alternativa de la relevancia de la inflación pasada es que indica que hay rigideces importantes o indización de precios y contratos. Idealmente, para capturar las expectativas, se debería incluir un valor explícito para esta variable. No obstante, este tipo de información no existe en muchos países de mercados emergentes.
} 


\section{La Inflación en México}

Durante el período 1980-2001, la inflación promedio resultó alta en México (41.8\%) y también volátil (desviación estándar de 38.9\%). Como puede observarse en la Gráfica 1, la inflación se relaciona positivamente con la depreciación del tipo de cambio nominal. En efecto, la inflación generalmente declinó en períodos de estabilidad y creció fuertemente en respuesta a las grandes devaluaciones asociadas con las crisis de balanza de pagos de 1982 y 1994 y la relacionada con la caída del precio del petróleo en 1986.

\section{Gráfica 1. Tasas de Crecimiento Anual Nominal del INPC, del Tipo de Cambio y de los Salarios Medios Manufactureros Período 1980-2001}

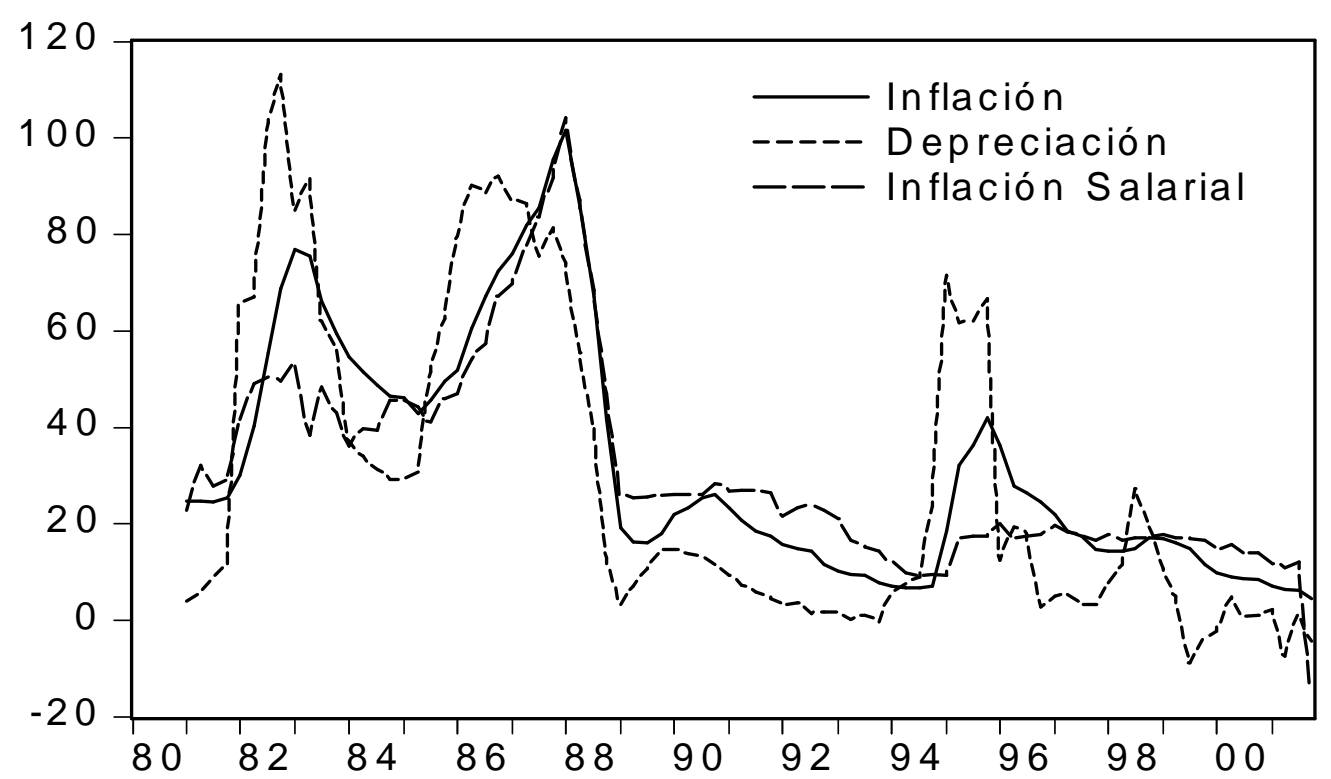

Mientras que la tasa de depreciación está positivamente relacionada con la inflación, ésta última ha sido menor que aquélla cuando las devaluaciones han sido pronunciadas. Sin embargo, ha permanecido por arriba de la tasa de depreciación cuando los movimientos del tipo de cambio fueron pequeños. La razón de esta asimetría radica en el comportamiento de los salarios nominales. En la Gráfica 1 también se muestra la evolución de los salarios medios manufactureros. Estos han aumentado en tiempos de crisis de balanza de pagos, pero han caído en términos reales, probablemente limitando el incremento en la inflación. En períodos de estabilidad del tipo de cambio y de inflación declinante, los salarios nominales se desaceleran pero en un grado menor que la caída de la inflación, causando incrementos en los salarios reales que pueden también ayudar a explicar porqué la inflación ha permanecido por encima de la tasa de depreciación. Es probable que la evolución de los salarios se encuentre fuertemente determinada por la brecha de producto, un asunto a ser discutido más adelante. 
El patrón observado en la Gráfica 1 ha motivado mucho del trabajo relativo a la inflación en México, el cual se ha enfocado en estimar los efectos del tipo de cambio y de los salarios sobre la inflación (se comenta un modelo con estas variables llamado de markup, en la sección 3.1). Santaella (2001) analiza la relación entre los precios y el tipo de cambio real durante el período 1969-2000 excluyendo a los salarios para que se cumpla la condición de la paridad del poder de compra (o PPP por sus siglas en inglés). Esto implica que el pass-through final del tipo de cambio a los precios internos tiene que ser uno.

\section{Modelos de Inflación}

La evidencia presentada ha llevado a los responsables de la política económica a concentrarse en tres clases de modelos de inflación. El primero concibe la inflación como un fenómeno de empuje de costos en el contexto de un modelo de margen sobre costos (markup) de largo plazo. El segundo considera a la inflación primariamente como un fenómeno directamente causado por el desequilibrio monetario y trata de ligar variaciones en los agregados monetarios con las de los precios. Cabe enfatizar que aunque los otros modelos no incluyan alguna función del dinero entre las variables explicativas ello no implica en modo alguno que la inflación no sea un fenómeno monetario, sino que el mecanismo que conecta a los precios y al dinero no es tan directo como sugieren los modelos llamados "monetarios". La tercera clase de modelos visualiza la inflación como proveniente de factores reales, en particular desequilibrios entre la demanda y la oferta agregadas. ${ }^{11}$

El primer tipo de modelo ha sido aplicado principalmente en mercados emergentes, mientras que los otros dos han sido típica, pero no exclusivamente, referidos a países industrializados. Más adelante, se amplía la discusión sobre estas tres clases de modelos.

\subsection{Modelos de Sobrecosto (Markup)}

Los modelos que se sustentan en la determinación de los precios como un margen sobre el costo marginal (que por brevedad llamaremos de sobrecosto) están usualmente basados, explícita o implícitamente en un modelo del tipo desarrollado por de Brouwer y Ericsson (1998) para la economía australiana. ${ }^{12}$ En este tipo de modelos, el nivel de precios se encuentra determinado por los costos y una tasa de sobrecosto en la siguiente forma:

\footnotetext{
${ }^{11}$ Estas caracterizaciones no son mutuamente independientes. Por ejemplo, si el banco central tuviera una previsión perfecta y la política monetaria fuese perfectamente creíble, entonces las tasas de interés y el tipo de cambio se ajustarían para asegurar que la oferta y demanda agregadas fuesen siempre iguales. Así, desde esta perspectiva, el desequilibrio entre ambos agregados puede ser visto como un fenómeno monetario.

${ }^{12}$ Goodfriend (1997) discute los fundamentos teóricos del modelo de sobreprecio (markup).
} 


$$
P_{t}=\mu_{t}\left(W_{t}\right)^{\gamma_{w}}\left(E_{t} P^{*}\right)^{\gamma_{e}}
$$

donde $\mathrm{P}$ es el nivel de precios interno, $\mu$ es la tasa de sobrecosto, $\mathrm{W}$ son los salarios, E es el tipo de cambio nominal y $\mathrm{P}^{*}$ es el nivel de precios externo, por lo que EP* es una medición de los precios externos expresados en moneda local. La ecuación de precios anterior puede ser derivada del problema de maximización de una empresa que enfrenta una curva de demanda con pendiente negativa (la empresa podría ser un monopolista o podría estar enfrentando competencia monopolística). En este contexto, la empresa vende a un precio igual a una tasa de sobrecosto por arriba del costo marginal, donde el costo marginal está determinado por el precio de los insumos nacionales, capturados por los salarios internos, y por el precio de los insumos externos, reflejado en el nivel de precios del exterior.

Expresando la ecuación anterior en logaritmos, el nivel de precios interno es un promedio ponderado de los salarios nominales y de los precios externos (expresados en unidades de moneda local):

$$
p_{t}=\ln \left(\mu_{t}\right)+\gamma_{w} w_{t}+\gamma_{e}\left(e_{t}+p_{t}^{*}\right)+\varepsilon_{t}
$$

donde las letras minúsculas indican variables en forma logarítmica. La especificación anterior es la base para estimar una relación de largo plazo entre los precios internos, los salarios y los precios externos suponiendo que la tasa de sobrecosto es constante o que fluctúa alrededor de un valor de largo plazo dado.

En el corto y mediano plazos, sin embargo, podrían tener lugar importantes y persistentes fluctuaciones de la tasa de sobrecosto dependiendo de cuan rápidamente los fijadores de precios se ajustan a variaciones en los salarios o en los precios externos. Así, al estimar la inflación mensual o trimestral, es importante considerar una dinámica más compleja de la siguiente forma:

$$
\Delta p_{t}=\alpha_{p} \Delta p_{t-1}+\alpha_{w} \Delta w_{t}+\alpha_{e} \Delta\left(e_{t}+p_{t}^{*}\right)-\delta\left(p_{t-1}-\gamma_{w} w_{t-1}+\gamma_{e}\left(e_{t-1}+p^{*}{ }_{t-1}\right)\right)+v_{t}
$$

donde el término rezagado captura la inercia inflacionaria, las variaciones contemporáneas en los salarios y en los precios externos se incluyen para capturar ajustes inmediatos y, finalmente, se incorpora un término de corrección de error que intenta capturar las desviaciones de la relación de largo plazo establecida en la ecuación (2).

El modelo de sobrecosto ha sido aplicado a México por Pérez-López (1996) y Garcés (1999). La especificación empleada por Pérez-López es una regresión puramente en tasas de variación para el período 1981-1995, mientras que Garcés incluye variables adicionales tales como la tasa de crecimiento de los precios administrados por el sector público, una medición simple de la brecha de producto y el término de corrección de error correspondiente para el período 1985- 
1998. ${ }^{13}$ Estas especificaciones de forma reducida tienden a ser una buena representación del proceso inflacionario en México, lo cual concuerda con la evidencia de la Gráfica 1.

Otros ejemplos del uso de modelos de sobrecosto para analizar o predecir la inflación - en adición a de Brouwer y Ericsson (1998) para Australia- incluyen Kenny y McGettigan (1999) para Irlanda, García y Restrepo (2001) para Chile, y Springer y Kfoury (2002) para Brasil. Estos son particularmente relevantes porque se refieren a economías pequeñas y abiertas o latinoamericanas. En el caso particular de los países latinoamericanos, los modelos de sobrecosto han tenido resultados excelentes en términos de su ajuste dentro y fuera de la muestra de las series históricas. Sin embargo, los recientes cambios de régimen en Brasil y Chile con la adopción de esquema de objetivos de inflación y tipos de cambio flexibles parecen haberse traducido en inestabilidad de los parámetros. $^{14}$ Dado que un cambio de régimen de política similar ha ocurrido en México, es de particular importancia evaluar modelos alternativos para considerar la posible inestabilidad en este tipo de especificación.

\subsection{Modelos Monetarios}

Los modelos monetarios están basados en la idea de que variaciones en el nivel general de precios son la respuesta de la economía a un desequilibrio monetario. Estos tipos de modelos son congruentes con el paradigma de "dinero activo," donde el dinero es visto como una parte activa del mecanismo de transmisión y que su exceso provoca inflación. ${ }^{15}$ Una situación de desequilibrio monetario existe si la cantidad de dinero en la economía se encuentra por arriba (debajo) de lo que el público desea mantener. Si esto ocurre, los modelos monetarios predicen que el nivel de precios aumentará (caerá) para reestablecer el equilibrio entre la oferta y la demanda de dinero. Así, un exceso de oferta de dinero puede traducirse en presiones inflacionarias de la misma manera que un exceso de demanda por bienes lo hace. El desequilibrio monetario típicamente se mide utilizando la brecha de dinero, la cual es la diferencia entre la oferta observada de dinero y la demanda de dinero de largo plazo estimada. De este modo, de acuerdo a los modelos monetarios, una brecha de dinero positiva (donde el acervo de dinero excede la demanda de dinero de largo plazo) debería estar asociada con presiones inflacionarias crecientes.

${ }^{13}$ Garcés (1999) encuentra un coeficiente de largo plazo de 0.63 para el nivel de precios externo y de 0.36 para los salarios.

${ }^{14}$ En particular, hay evidencia sólida de que el pass-through ha caído bastante tanto en Brasil como en Chile. Podría ser el caso de que algo similar haya ocurrido en México, pero la estabilidad de la cotización peso/dólar durante los últimos cuatro años implica que las pruebas de cambio estructural tengan muy bajo poder explicativo.

${ }^{15}$ Esto contrasta con el paradigma de "dinero pasivo" donde al dinero no se le asigna un papel causal en el mecanismo de transmisión y se concibe como respondiendo a cambios en los precios, el producto y las tasas de interés. El dinero en este contexto es visto como indicador de la actividad económica. Para una revisión de los dos paradigmas, ver Engert y Selody (1998). 
Los modelos monetarios han sido aplicados extensivamente en países industrializados. Un ejemplo reciente de ello es Altimari (2001) quien evalúa el desempeño de varios modelos monetarios de inflación para la zona del euro para el período 1980-2000. Su estudio sugiere que los agregados monetarios y crediticios contienen información significativa e independiente para predecir la inflación en esa zona, especialmente en horizontes de mediano plazo. Kasumovich (1996) y Fung y Kasumovich (1998) encuentran apoyo para la hipótesis del "dinero activo" en su análisis con vectores autorregresivos estructurales de los países industrializados más importantes. Sus resultados sugieren que un desequilibrio monetario surge y persiste a consecuencia de una perturbación de política monetaria, y se cierra conforme los precios se ajustan a lo largo de algunos años.

En otro trabajo, Hendry (1995) estimó un modelo de vector autorregresivo de corrección de error (VECM) para la demanda de M1 en Canadá, y Armour et al. (1996) y Engert y Hendry (1998) encontraron que este VECM producía buenos pronósticos de inflación de uno a dos años. ${ }^{16}$ Igualmente, Hallman, Porter y Small (1991) estimaron una relación de largo plazo entre el dinero y los precios en los Estados Unidos. Ellos encuentran que la velocidad del dinero es un proceso estacionario y que la brecha entre el nivel de precios observado y el nivel de precios dado por la velocidad de largo plazo del dinero tiene un significativo poder explicativo de la inflación.

Los modelos monetarios han sido también aplicados a mercados emergentes. Por ejemplo, Jonsson (1999) estima un VECM estructural para Sudáfrica que incluye los precios internos, el producto, el tipo de cambio nominal, los precios externos y la tasa de interés interna. Ese autor encuentra una relación de largo plazo estable entre estas variables y muestra que un incremento en la oferta de dinero eleva los precios internos, aunque este efecto es de alguna forma contrarrestado por un incremento en las tasas de interés internas. En otro ejemplo, Callen y Chang (1999) estiman dos modelos de inflación para India (uno basado en el enfoque monetario y el otro usando la brecha de producto). Ellos concluyen que los agregados monetarios contienen la mejor información sobre la inflación futura y que la brecha de producto no es una variable explicativa importante.

Los modelos monetarios necesitan ser usados con precaución. La innovación financiera puede cambiar la demanda de dinero (especialmente las definiciones más estrechas), introduciendo inestabilidad en la relación entre el dinero y la inflación. Más aún, la elasticidad tasa de interés del dinero en el corto plazo puede ser alta, llevando a los responsables de política a creer que un incremento de las tasas de interés suficiente para llevar al dinero de regreso a su objetivo podría ser

\footnotetext{
${ }^{16}$ Los coeficientes de la desviación del dinero de su equilibrio de largo plazo implican que cuando el agregado monetario está por arriba de su demanda de largo plazo, el dinero decrecerá y los precios se incrementarán para restaurar el equilibrio de largo plazo. Los efectos de la desviación sobre el producto y las tasas de interés no son significativos, indicando la exogeneidad débil de estas variables. La implicación de los resultados es que todo el ajuste para regresar la economía al equilibrio monetario proviene de movimientos de los precios y del dinero.
} 
demasiado pequeño para reducir el gasto y las presiones inflacionarias. ${ }^{17}$ No obstante, según observa King (2002), hay buenas razones para desarrollar modelos basados en agregados monetarios. Primero, como es bien sabido, los modelos basados en el crecimiento monetario tienen una capacidad limitada para pronosticar la inflación de corto plazo, pero se desempeñan mejor como indicadores de presiones inflacionarias de largo plazo. ${ }^{18}$ Segundo, en un contexto de inflación baja, la probabilidad de caer en una trampa de liquidez con tasas de interés nominal cercanas a cero y deflación implica que una expansión de la base monetaria podría ser la única forma de relajar las condiciones monetarias aún más. Así, es conveniente analizar el efecto de las variaciones en los agregados monetarios independientemente de su efecto sobre las tasas de interés. Finalmente, al enfocarse solamente en otros determinantes de las condiciones monetarias de la economía, hay un riesgo de ignorar un importante componente del mecanismo de transmisión.

\subsection{Curvas de Phillips}

Desde que fue esbozada por Phillips (1958) en el artículo seminal, la curva de Phillips ha sido usada extensivamente como un marco de referencia para explicar y predecir la inflación en los países industrializados. En su forma original, la curva de Phillips estipulaba una relación entre la tasa de variación en los salarios nominales y el inverso de la tasa de desempleo, donde esta última era usada como una medida aproximada del exceso de demanda. ${ }^{19}$ Así, un descenso de la tasa de desempleo implicaba un incremento del exceso de demanda, el cual presionaba hacia arriba a los salarios nominales. En la década que siguió a su aparición, la curva de Phillips experimentó varias modificaciones. Por ejemplo, el inverso de la tasa de desempleo fue reemplazado por las brechas de desempleo o de producto como una aproximación al exceso de demanda. ${ }^{20}$ En adición, el papel de las expectativas para incidir en los cambios salariales fue reconocido y las expectativas inflacionarias fueron incorporadas. ${ }^{21}$ Finalmente, se transformó de una inflación salarial a una ecuación de inflación de precios. $^{22}$

${ }^{17}$ Para una discusión de porque Canadá abandonó los objetivos de agregados monetarios y cual es el papel actual de éstos en la formulación de la política, ver Freedman (2000).

${ }^{18}$ Esto, desde luego, supone que no hay innovaciones financieras profundas en el período dado.

${ }^{19}$ Phillips (1958) encontró una relación estable entre la inflación salarial y la tasa de desempleo utilizando datos para el Reino Unido para el período de 1861 a 1957.

${ }^{20}$ La brecha de desempleo es la diferencia entre las tasas natural y observada, donde la tasa natural se define como aquélla que prevalece cuando las expectativas se han realizado e incorporado completamente en todos los precios y salarios, y la inflación no tiende a acelerarse o desacelerarse. La brecha de producto es la desviación del producto observado respecto de su potencial donde este último es el nivel que es compatible con una inflación estable dado el acervo de capital. El desempleo y la brecha de producto están ligados a través de la ley de Okun, la cual relaciona variaciones en el desempleo con cambios en el producto.

${ }^{21}$ Friedman (1968) generalmente recibe el crédito de haber sido el primero en enfatizar la importancia de incluir las expectativas inflacionarias en la curva de Phillips. Como él notó, a las empresas y a los trabajadores les conciernen los salarios reales y no los nominales; en otras palabras, ellos observan a la tasa de cambio de los salarios nominales menos la inflación esperada.

${ }^{22}$ Las dos especificaciones son congruentes si se supone que los precios se fijan aplicando una tasa de sobrecosto constante sobre el costo salarial unitario. 
Aunque varias formas de la curva de Phillips se usan en la práctica, la mayoría de ellas puede ser interpretada como un modelo "triangular" de la inflación—un término que Gordon (1997) acuñó para resumir la dependencia de la inflación en este contexto en tres determinantes básicos: inercia, demanda y oferta. Este marco de la curva de Phillips se denomina algunas veces como la curva de Phillips tradicional, para distinguirla de la nueva. ${ }^{23}$ La nueva curva de Phillips es similar en espíritu a la versión tradicional en tanto que supone que la inflación varía positivamente con el sector real de la actividad económica. Proponentes de la nueva curva de Phillips señalan que esta formulación es más apropiada dado que se deriva explícitamente de un modelo de fijación traslapada de precios por empresas en competencia monopolística, y de esta forma tiene fundamentos teóricos más sólidos. ${ }^{24}$ Vale la pena notar, sin embargo, que al imponer ciertas restricciones sobre la tecnología y la estructura del mercado laboral, y en la vecindad del estado estacionario, el costo marginal real es proporcional a la brecha de producto.$^{25}$

La persistente popularidad de la curva de Phillips tradicional es probablemente debida en parte a su relativo éxito como una herramienta de pronóstico. Como apuntan Stock y Watson (1999), "como una herramienta para predecir inflación, [la curva de Phillips] es ampliamente considerada estable, confiable y adecuada, al menos comparada con las alternativas." Duguay (1994) estimó una curva de Phillips para Canadá para el período 1968Q4 a 1990Q4 y encontró que esa ecuación era razonablemente exitosa para explicar variaciones en la inflación canadiense sobre dicho período. Trabajos posteriores en esta área se han enfocado en la forma de la curva de Phillips de corto plazo y en la asimetría entre las brechas de producto positivas y negativas y sus efectos sobre la inflación. ${ }^{26}$

En años recientes, las curvas de Phillips tradicionales han tenido más dificultades en predecir la inflación. En Canadá, por ejemplo, la inflación cayó de 4\% en 1990 a 2\% entre 1993 y 1995. Sin embargo, las curvas de Phillips tradicionales habrían predicho una deflación al final de ese período, dado el vasto monto de exceso de oferta en la economía canadiense. Más aún, a finales de los noventa, la inflación en los Estados Unidos fue relativamente baja dado el rápido crecimiento del producto. Estas experiencias han llevado a algunos observadores a predecir "la muerte de la curva de Phillips." ${ }^{27}$ Los economistas han adoptado dos estrategias para tratar con la aparente falla de la relación tradicional. Por un lado, se hicieron ajustes a la curva de Phillips

\footnotetext{
${ }^{23}$ Para una revisión completa de la literatura de la nueva curva de Phillips, ver Goodfriend y King (1997).

${ }^{24}$ En estos modelos, las empresas en competencia monopolística y sus trabajadores fijan un contrato salarial para $\mathbf{J}$ períodos. Las negociaciones salariales se supone que se traslapan en el tiempo, con $1 / \mathrm{J}$ de los contratos fijados en cada período. Las empresas entonces determinan los precios con base en una tasa de sobrecosto sobre una combinación de costo marginal realizado y esperado. La naturaleza traslapada del proceso de fijación de precios nominales en la economía significa que los precios responderán gradualmente a cambios en el producto. En estos modelos, los rezagos de precios y salaries nominales son importantes porque reflejan el ajuste gradual a las perturbaciones.

${ }^{25}$ Ver Gali, Gertler y López-Salido (2001), y las referencias ahí citadas, para más sobre este punto.

${ }^{26}$ Para una revisión de este trabajo ver Macklem (1997).

${ }^{27}$ Gali, Gertler, y López-Salido (2001), p.5.
} 
tradicional para considerar cambios estructurales. ${ }^{28}$ Por otro, un enfoque más estructural fue aplicado al modelarse la relación entre el desequilibrio laboral y la inflación, el cual ha llevado a la literatura de la Nueva Curva de Phillips.

En años recientes, ha habido aplicaciones de la curva de Phillips tradicional a países de mercados emergentes. Coe y McDermott (1999) estiman curvas de Phillips basadas en brechas de producto para trece países asiáticos. Ellos encuentran que para once de los trece países, la brecha de producto es un determinante significativo de la inflación, aún cuando otras variables tales como mediciones del desequilibrio monetario son incluidas en las ecuaciones. Simone (2000) estima curvas de Phillips que varían con el tiempo para Chile. El encuentra que aunque el modelo que incluye los objetivos de inflación preanunciados presenta alguna autocorrelación, se desempeña mejor que los modelos que excluyen esta variable en ejercicios de pronóstico.

\section{Tres Modelos de Inflación para México: Resultados de la Estimación dentro de la Muestra}

En esta sección, se presentan las especificaciones de los tres modelos de inflación que se aplican a México y se compara su desempeño dentro de la muestra. Cada modelo se estima usando datos trimestrales para el período 1983Q1 a 2001Q4. ${ }^{29}$ En todos los casos se utiliza la inflación de trimestre a trimestre como la variable dependiente. ${ }^{30}$ En la primera gráfica en el Apéndice 1 se presenta la trayectoria de esta variable durante el período de muestra.

\subsection{Modelo de Sobrecosto}

Se utiliza la siguiente especificación para el modelo de sobrecosto, la cual se basa en Garcés (1999):

$$
\Delta p_{t}=\alpha_{c}+\alpha_{p} \Delta p_{t-1}+\alpha_{w} \Delta w_{t}+\alpha_{e} \Delta\left(e_{t}+p_{t}^{*}\right)-\delta\left(p_{t-1}-\gamma_{w} w_{t-1}+\gamma_{e}\left(e_{t-1}+p_{t-1}^{*}\right)\right)+v_{t}
$$

${ }^{28}$ Por ejemplo, Filion y Leonard (1997) introdujeron un proceso de cambio de régimen de Markov en una curva de Phillips tradicional y aplicaron este modelo a la inflación canadiense. Encontraron que al incorporarse el cambio de régimen mejoran considerablemente los pronósticos. En otro ejemplo, Kichian (2001) estimó una curva de Phillips para Canadá utilizando un marco estado-espacio en el cual los parámetros del modelo varían a lo largo del tiempo. Al reestimar la relación tradicional con los parámetros cambiantes en el tiempo, las expectativas de inflación se modelaron como condicionalmente endógenas. Lo anterior mostró que las expectativas de inflación y el passthrough fueron reducidos en Canadá durante los noventa y que el coeficiente para la brecha de producto fue la mitad de su valor entre 1978-1988.

${ }^{29}$ Se usa este período de muestra debido a la disponibilidad de datos. En particular, la serie trimestral del PIB en México comienza en 1980.

${ }^{30}$ Debe señalarse que la inflación se mide con base en el índice de precios al consumidor al final de cada trimestre. 
Los parámetros de largo plazo pueden ser estimados por casi cualquier método de cointegración con resultados muy similares. Sin embargo, por simplicidad y por el hecho de que los parámetros son reestimados recursivamente en el ejercicio de pronóstico, se utilizó el marco del modelo de corrección de error no restringido. Los resultados para toda la muestra se reportan en la primera columna del Cuadro 2.1 del apéndice y un análisis detallado de la relación de largo plazo puede ser visto en Garcés (1999). El modelo en ese cuadro contiene una constante, el término de corrección de error rezagado $\left(E C_{\mathrm{t}-1}\right)$, la inflación rezagada $\left(\Delta p_{\mathrm{t}-1}\right)$, las tasas de crecimiento contemporáneo de los salarios $\left(\Delta w_{\mathrm{t}-1}\right)$ y los precios externos $\left(\Delta e p e u_{\mathrm{t}-1}\right)$ y una variable dicotómica para el segundo trimestre de 1988 para obtener residuales normales. Los coeficientes estimados para todas las variables son significativos a cualquier nivel razonable. La bondad de ajuste es la más alta en todos los modelos del Cuadro 2.1, un hecho que se verá reflejado en el desempeño del pronóstico. El único problema detectado por los estadísticos de evaluación (debajo de los estimados) es el elevado estadístico RESET. Esto indica un problema con la especificación lineal elegida, el cual no se intentó resolver aquí.

\subsection{Modelo de Brecha de Dinero.}

El modelo de brecha de dinero que se utiliza tiene la siguiente forma:

$$
\Delta p_{t}=\alpha+\beta_{1} \Delta p_{t-1}+\beta_{2} \text { moneygap }_{t-1}+\varepsilon_{t}
$$

donde la variable clave es la brecha de dinero moneygap $t$, , la medida de desequilibrio monetario que se seleccionó. La brecha de dinero se obtiene de la siguiente demanda de dinero de largo plazo, donde el logaritmo del dinero real es una función lineal de una variable de escala, representada en este caso por el índice de la producción industrial $(\mathrm{y})^{31}$, y del costo de oportunidad del dinero (la tasa de interés $i$ ):

$$
m-p=c+\gamma y+\pi i
$$

Para el caso de México, una función de demanda de dinero de largo plazo puede ser estimada para casi cualquier definición de dinero, aunque se decidió usar billetes y monedas en este documento. Ello, porque es el agregado que el Banco de México usó como objetivo intermedio y porque es el más cercanamente relacionado a la inflación y a la actividad económica. ${ }^{32}$ De nueva cuenta, los parámetros de largo plazo pueden ser obtenidos con cualquier método. En el cuadro 1 se muestran los estimados de esta demanda de largo plazo para cada uno de diversos agregados

\footnotetext{
${ }^{31}$ Se procede de esta manera para hacer los resultados comparables con los obtenidos en Garcés (2001), aunque éstos no varían en ninguna manera significativa al utilizarse otras medidas tales como el PIB trimestral o el consumo privado.

${ }^{32}$ Ver Garcés (2002).
} 
monetarios. Todas las ecuaciones de largo plazo se obtienen para saldos monetarios reales (esto es, el agregado monetario dividido entre el índice de precios al consumidor). El método de estimación usado es FM-LS de Phillips y Hansen (1990). Las columnas 3 a 6 contienen los estadísticos propuestos por Hansen (1992) para probar la estabilidad de los parámetros de largo plazo junto con los valores $\mathrm{p}$ correspondientes.

\section{Cuadro 1}

\section{Funciones de Demanda de Dinero de Largo Plazo y Pruebas de Estabilidad}

\begin{tabular}{|c||c|l|}
\hline \multicolumn{1}{|c||}{ Agregado } & \multicolumn{2}{|c|}{ Elasticidades de Largo Plazo* } \\
\cline { 2 - 3 } & $\mathrm{I}$ & \multicolumn{1}{|c|}{$\mathrm{Y}$} \\
\hline myb-p & -0.353 & 0.566 \\
& $(0.054)$ & $(0.086)$ \\
\hline $\mathrm{m} 1-\mathrm{p}$ & -0.960 & 1.100 \\
& $(0.212)$ & $(0.332)$ \\
\hline $\mathrm{m} 2-\mathrm{p}$ & -0.281 & 1.284 \\
& $(0.174)$ & $(0.272)$ \\
\hline $\mathrm{m} 3-\mathrm{p}$ & -0.214 & 1.369 \\
& $(0.082)$ & $(0.128)$ \\
\hline $\mathrm{m} 4-\mathrm{p}$ & -0.147 & 1.891 \\
& $(0.112)$ & $(0.175)$ \\
\hline
\end{tabular}

\begin{tabular}{|l|c|l|}
\hline \multicolumn{3}{|c|}{ Pruebas de Estabilidad $* *$} \\
\hline LC & F prom. & F. sup \\
\hline 0.410 & 3.940 & 10.033 \\
$\mathrm{p}=0.162$ & $\mathrm{p}=0.200$ & $\mathrm{p}=0.200$ \\
\hline 0.362 & 4.234 & 18.028 \\
$\mathrm{p}=0.200$ & $\mathrm{p}=0.186$ & $\mathrm{p}=0.014$ \\
\hline 0.210 & 2.489 & 9.631 \\
$\mathrm{p}=0.200$ & $\mathrm{p}=0.200$ & $\mathrm{p}=0.200$ \\
\hline 0.534 & 4.744 & 8.318 \\
$\mathrm{p}=0.081$ & $\mathrm{p}=0.135$ & $\mathrm{p}=0.200$ \\
\hline 0.748 & 5.706 & 11.559 \\
$\mathrm{p}=0.028$ & $\mathrm{p}=0.071$ & $\mathrm{p}=0.187$ \\
\hline
\end{tabular}

*Errores estándar en paréntesis

$* * \mathrm{p}=$ Valor $\mathrm{p}$ del estadístico

Los signos de los parámetros de largo plazo son los correctos y las magnitudes razonables. Los valores $\mathrm{p}$ en la mayor parte de los casos están arriba de 0.05 , indicando que la hipótesis de parámetros constantes no se puede rechazar. Con los estimados de billetes y monedas (myb-p) se construye la brecha de dinero moneygap: $:^{33}$

$$
\text { moneygap }=m-p-0.566 y+0.353 i
$$

Este es el término de corrección de error en una demanda de dinero de corto plazo. En una ecuación donde la inflación es la variable independiente proporciona el canal para que el desequilibrio monetario pueda impactar la dinámica de las variaciones de precios. En el caso que se analiza aquí, esta variable no fue muy significativa en todas las especificaciones que se intentaron y esto es también lo que ocurre para los demás agregados. Los resultados se presentan en la segunda columna del Cuadro 2.1 y muestran que la brecha de dinero rezagada no tiene mucho poder explicativo para la inflación; esto se reflejará en el pobre desempeño predictivo de este

\footnotetext{
${ }^{33}$ Ninguna conclusión de este artículo se altera si se utiliza un agregado alternativo.
} 
modelo. Corrigiendo el problema de no normalidad de los residuales con variables dicotómicas hace que la brecha monetaria sea aún menos significativa.

\subsection{La Curva de Phillips}

Se utiliza una especificación de la curva de Phillips tradicional que relaciona la inflación de precios a las expectativas inflacionarias, a alguna medida del desequilibrio real y a una variable que captura las variaciones en los precios de importación dado que México es una economía relativamente abierta. Suponiendo que las expectativas se forman adaptativamente, de tal forma que la inflación rezagada pueda usarse como una aproximación de las expectativas inflacionarias y que la relación es lineal, se obtiene la siguiente especificación:

$$
\pi_{t}=\alpha+\beta_{1}(L) \pi_{t}+\beta_{2}(L) g a p_{t}+\beta_{3}(L) \Delta e_{t}+\varepsilon_{t}
$$

donde $\pi_{\mathrm{t}}$ es la tasa de inflación, $L$ es el operador de rezagos, $\Delta$ e es la tasa de depreciación, gap $p_{\mathrm{t}}$ es la brecha de producto y $\varepsilon_{\mathrm{t}}$ es el término de error en el momento t. En términos de las variables explicativas, se utilizó una medición de la brecha de producto obtenida de un VAR estructural (que se discute más adelante) la variación trimestral del tipo de cambio bilateral peso-dólar. ${ }^{34}$ Las gráficas que muestran la evolución de estas variables se presentan en el apéndice.$^{35}$

La especificación en la ecuación 7 presupone que la inflación, la brecha de producto y la depreciación nominal son todas variables estacionarias. Se condujeron pruebas de raíz unitaria en todas las variables para confirmar que éste es el caso. Los resultados de la prueba de Dickey-Fuller aumentada (ADF) y de Phillips-Perron (PP) se muestran en el Apéndice 3. Para ambas pruebas, la hipótesis nula es que las serie en cuestión contienen una raíz unitaria; así, un rechazo de la hipótesis nula sugiere que la series es estacionaria. Los resultados de la prueba para la brecha de producto no son ambiguos y sugieren que esta serie es estacionaria. Para las otras dos variables, los resultados de las pruebas no son concluyentes. En el caso de la inflación, todas las pruebas excepto para ADF sin tendencia sugieren una serie estacionaria. En el caso de la depreciación nominal, la prueba ADF sugiere una raíz unitaria mientras que PP apunta a una serie estacionaria. Con base en estos resultados y en percepciones a priori, se supuso que tanto la inflación como la tasa de depreciación son estacionarias durante el período de muestra.

La brecha de producto para México se estima por medio de un VAR estructural, una metodología desarrollada por Blanchard y Quah (1989), entre otros, y aplicada a México por De

\footnotetext{
${ }^{34}$ La tasa de depreciación trimestral se basa en el tipo de cambio que prevalece al final de cada trimestre.

${ }^{35}$ Todas las series utilizadas no se han ajustado estacionalmente aunque se incluyen variables dicotómicas estacionales en las ecuaciones de corto plazo.
} 
Serres, Guay y St-Amant (1995). ${ }^{36}$ En esta metodología se supone que el producto es determinado por una combinación de perturbaciones de demanda y oferta, y que al identificarlas se puede descomponer al producto en sus partes permanente y transitoria. Se obtiene estimando un VAR en el cual otras variables, que también se supone que están determinadas por las mismas perturbaciones, se añaden al sistema para ayudar a identificar las perturbaciones de oferta y demanda. Adicionalmente, algunas restricciones sobre la evolución de largo plazo de estas variables en respuesta a perturbaciones son necesarias para identificar a estas perturbaciones; las restricciones anteriores están basadas en la teoría macroeconómica .

De Serres, Guay y St-Amant (1995) estimaron un VAR de 3 variables para México para el período 1965-1994 el cual incluía el precio del petróleo, la producción industrial (como una aproximación al producto global) y un agregado monetario, todo en primeras diferencias. ${ }^{37}$ Para identificar las perturbaciones del petróleo y otras perturbaciones de oferta y demanda, los autores suponen que las perturbaciones de demanda no tienen un efecto permanente sobre el producto en el largo plazo y que el precio del petróleo es exógeno a la economía mexicana. Los autores mencionados después calculan el producto potencial al añadir las perturbaciones del petróleo y otras perturbaciones de oferta a la parte permanente del producto.

Se aplicó en este trabajo la misma metodología, pero se utilizó el precio del petróleo, el PIB real y el tipo de cambio real, todos en primera diferencia, y se estimó el sistema para el período 1980-2001..$^{38}$ Se optó por el tipo de cambio real en lugar de un agregado monetario porque se consideró que ésta es una variable rica en información para una economía abierta como México. La percepción a priori era que los movimientos del tipo de cambio real reflejarían muchas perturbaciones de oferta y demanda importantes para México y, en consecuencia, proporcionarían información útil para llegar a distinguir entre perturbaciones de demanda y oferta. En particular, podría ayudar a considerar las perturbaciones al financiamiento externo que se han traducido en severas crisis de balanza de pagos en México.

Además, se intentaron diferentes especificaciones del VAR, incluyendo sistemas de 3 a 5 variables usando, además, un agregado monetario y la tasa de desempleo. Se encontró que el sistema de 3 variables con el tipo de cambio real proporcionaba el perfil de brecha de producto más razonable para el período de muestral.

\footnotetext{
${ }^{36}$ Una descripción más detallada de esta metodología se encuentra en el Apéndice 4.

${ }^{37}$ Las series se diferenciaron para asegurar estacionariedad.

${ }^{38}$ Se aplicaron pruebas de raíz unitaria para las series en primeras diferencias a fin de garantizar que éstas fueran estacionarias. Como se muestra en el Cuadro 3.1 en el Apéndice 3, los resultados de estas pruebas sugieren que las variables en niveles son I(1) y así, una vez diferenciadas, son estacionarias. En adición, como se muestra en el Cuadro 4.3, se probó si las series en niveles estaban cointegradas utilizando el método de Johansen y no se encontró evidencia de que así ocurriera.
} 
Efectivamente, como se señala en el Apéndice 1, la brecha de producto estimada para las dos décadas pasadas es congruente con los conocidos episodios de auge y recesión que México ha experimentado durante este período. Se pueden identificar cuatro contracciones mayores en este período que estuvieron caracterizadas por brechas de producto negativas, y en algunos casos sostenidas: la prolongada contracción de principio de los ochenta que ocurrió como resultado de la crisis de la deuda, la desaceleración económica de 1993, la crisis económica que siguió la devaluación del peso a mitad de los noventa, y el leve declive de 1998-1999 que fue causado principalmente por una caída de los precios del petróleo y por un deterioro del sentimiento del mercado después de la moratoria rusa. En contraste, el auge que precedió a la crisis del peso de 1994 se muestra con una serie de brechas de producto positivas y amplias.

Al estimarse la ecuación 7 para México, se comenzó con cuatro rezagos para cada una de las variables explicativas y se encontró que solamente el primer rezago de la inflación, el segundo rezago de la brecha de producto y el primer rezago de la depreciación fueron estadísticamente significativos. Dado que el objetivo de este artículo es examinar modelos que puedan ser usados tanto para explicar como para pronosticar la inflación, se decidió usar esta especificación parsimoniosa. ${ }^{39}$ Además de la inclusión de los valores rezagados, también se intentaron especificaciones con valores contemporáneos de la brecha de producto y de la tasa de depreciación, pero solamente la última de éstas fue estadísticamente significativa.

Se reportan los resultados de la estimación para la versión parsimoniosa de la curva de Phillips en la tercera columna del Cuadro 2.1 del Apéndice 2. Como se muestra en el cuadro, la curva de Phillips hace un buen trabajo para explicar los movimientos de la inflación mexicana durante las dos décadas pasadas. Efectivamente, la $\mathrm{R}$ cuadrada ajustada es alta y todos los coeficientes son estadísticamente significativos; adicionalmente, tienen el signo correcto y son de una magnitud razonable. Los coeficientes estimados de la curva de Phillips son generalmente congruentes con los supuestos previos. Por ejemplo, el coeficiente de la inflación rezagada es aproximadamente 0.6. Esto indica que un incremento de $1 \%$ en la inflación del trimestre anterior se convertirá en $0.6 \%$ de inflación en el trimestre actual. El coeficiente de la brecha de producto es positivo e implica que un aumento de $1 \%$ en el mismo presionará la inflación a subir $0.4 \%$ en el corto plazo. El efecto contemporáneo de la depreciación se estima en 0.34.

En adición a los resultados reportados en el Cuadro 2.1 del apéndice, también se incluye la estimación de un modelo AR(1). Este modelo será usado como un estándar mínimo para los tres modelos más elaborados. La especificación puede parecer muy sencilla pero los rezagos adicionales e incluso los términos MA no son significativos. La mejora de los modelos propuestos con respecto al simple modelo autorregresivo es notable, con la excepción del modelo monetario

\footnotetext{
${ }^{39}$ En otras palabras, incluir rezagos adicionales de las variables explicativas cuando no añaden información al modelo no es particularmente útil.
} 
que solamente experimenta una mejoría marginal. El modelo que parece ser mejor es el de sobrecosto ya que tiene el coeficiente de bondad de ajuste más alto y el menor error estándar de la regresión (EER). A continuación se examina el desempeño predictivo de los tres modelos.

\section{Comparación del Desempeño Predictivo}

En esta sección se examina el desempeño de los tres modelos en pronósticos fuera de muestra; se comparan entre sí y con el modelo AR(1). Para hacer esto se estiman todos los modelos de modo recursivo comenzando con 1983Q1-1996Q4 como período inicial, aumentando una observación más cada trimestre y generando un nuevo pronóstico; posteriormente se acumula la inflación pronosticada para los siguientes cuatro trimestres. Se lleva a cabo lo anterior utilizando tanto valores observados como pronosticados de las variables explicativas. ${ }^{40}$ Los valores pronosticados de las series utilizadas son aquéllos que habrían estado disponibles al momento de hacer el pronóstico. Así, en el caso cuando el período de estimación terminó en 1996Q4 y el período de pronóstico comenzó en 1997Q1, se utilizó la información que habría estado disponible en dicho trimestre. Se necesitan valores pronosticados de las variables explicativas en todos los modelos excepto en el de la inflación rezagada, lo cual puede ser obtenido de las propias ecuaciones de pronóstico. Los valores pronosticados para las variables explicativas fueron derivados de una combinación de modelos y pronósticos del mercado. ${ }^{41}$

El desempeño fuera de la muestra fue comparado en los Cuadros 2.2 y 2.3 del Apéndice 2 usando tres diferentes medidas: la raíz del error medio cuadrático (RMSE), el error absoluto medio(MAE) y el error medio(ME). Los resultados sugieren que el modelo con el mejor desempeño basado en estos ejercicios es el de sobrecosto, y esto ocurre sin importar si los valores pronosticados de las variables explicativas fueron los realizados o los pronosticados. La curva de Phillips se desempeña mejor que el modelo de la brecha de dinero y que el modelo AR1 utilizando los valores observados de las variables explicativas. Sin embargo, se desempeña peor cuando se utilizan los valores pronosticados de las mismas.

\section{Implicaciones de los Resultados para México en un Ambiente de Baja Inflación}

Los resultados presentados en las secciones 4 y 5 sugieren que, aun en el período más reciente, los modelos que incorporan al tipo de cambio se desempeñan bien en términos de explicar y pronosticar la inflación en México. Tanto el modelo de sobrecosto (markup) como la curva de

\footnotetext{
${ }^{40}$ En el caso de la brecha de producto, no hay valores realizados porque esta variable no es observable. La brecha de producto corresponde al escenario de información completa que se estima con un VAR estructural utilizando las variables observadas.
} 
Phillips se desempeñan mejor que los otros modelos porque en ambos el tipo de cambio tiene un papel prominente aun con una variable adicional en cada caso (salarios o la brecha de producto).

Las implicaciones de estos resultados para el ambiente actual de baja inflación observado en México no son obvias. Ello, porque las estimaciones han cubierto períodos de alta y baja inflación. Sin embargo, el desempeño de pronóstico de los modelos sugiere que el de sobrecosto ha sido más congruente en términos de producir errores más pequeños aun en el período más reciente. Esto no significa que otras variables diferentes al tipo de cambio no se volverán más importantes en el futuro sino que trabajo adicional será necesario para identificar sus efectos en un entorno de estabilidad.

Hay rezones de fondo para esperar un cambio en la importancia relativa de los diferentes determinantes basados en la observación de la historia mexicana previa. Como un ejemplo de esta afirmación, considérese el efecto de la inflación de los Estados Unidos sobre la inflación mexicana. En las ecuaciones presentadas en este trabajo, esta variable, siempre que ha sido necesario, se restringe a tener los mismos coeficientes de la depreciación del tipo de cambio o de lo contrario se vuelve no significativa. Sin embargo, se sabe que esta variable es importante porque fue necesaria para mantener la condición de PPP (el nivel de precios desde 1980 se ha incrementado en casi exactamente la misma proporción que los precios de los Estados Unidos expresados en pesos mexicanos) pero su efecto individual en el período de muestra es difícil de detectar porque el tipo de cambio ha sido muchas veces más variable. Si el mismo efecto es estimado para el periodo cuando el tipo de cambio se encontraba fijo en México (de 1954 a 1975), entonces el coeficiente de la inflación de los Estados Unidos es muy significativo a cualquier nivel propuesto. Algo similar ocurre con la brecha monetaria.

En lo que respecta a la brecha de producto, los lapsos en la historia mexicana cuando la actividad económica ha estado evidentemente arriba del potencial fueron pocos antes del período de muestra. Así, su efecto sobre la inflación es difícil de medir en ese caso también. Uno de estos episodios tuvo lugar al final de los setenta porque el gasto gubernamental se incrementó considerablemente bajo la expectativa de mayores ingresos petroleros. Muchos analistas consideraron entonces que una gran parte del impulso inflacionario en ese período provenía de una elevada demanda agregada (el tipo de cambio estaba fijo). Por estas razones se piensa que tan pronto como la variabilidad del tipo de cambio se estabilice a un nivel bajo, como ha ocurrido en los últimos años, el énfasis de la política monetaria se desplazará a otro tipo de perturbaciones.

\footnotetext{
${ }^{41}$ Se usaron los pronósticos del mercado producidos por el Consensus Forecasts para el tipo de cambio, los niveles de precios de México y los Estados Unidos, el precio del petróleo y el PIB mexicano.
} 


\section{Conclusiones}

Los resultados de estimación y pronóstico en este trabajo sugieren que la evolución del tipo de cambio continúa siendo un factor muy importante para explicar la inflación. En efecto, el modelo que se desempeña mejor es aquél que le asigna mayor importancia al tipo de cambio. La curva de Phillips hace un buen trabajo para explicar y pronosticar la inflación cuando se utilizan valores observados de las variables explicativas, pero no lo hace tan bien cuando se usan valores pronosticados de ellas. El modelo de la brecha de dinero no parece ser muy útil en su forma actual dado que es incapaz de superar a un simple modelo AR1. ${ }^{42}$ Sin embargo, valdrá la pena explorar si modelos monetarios más sofisticados se desempeñan mejor para explicar y pronosticar la inflación en México. Se deja esto para una investigación futura.

Como se señaló, la política monetaria por naturaleza propia es conducida en un ambiente caracterizado por la incertidumbre y el cambio tanto en países industriales como en desarrollo. La incertidumbre es probablemente mayor en un país como México, donde modificaciones significativas de su ambiente económico y político han tenido lugar durante las dos últimas décadas. Todo esto sugiere que podría haber un espacio para múltiples modelos de inflación en la conducción de la política monetaria mexicana. Con base en los resultados presentados en este artículo, parece ser que el modelo de sobrecosto (markup) y la curva de Phillips aumentada con el tipo de cambio podrían ofrecer visiones complementarias del contexto mexicano. Sin embargo, es evidente que en ambos modelos el tipo de cambio aún tiene un papel dominante. En la medida que la volatilidad de esta variable se ha reducido, es de esperarse un mayor papel para otras variables.

\footnotetext{
${ }^{42}$ No parece apropiado incluir la depreciación del tipo de cambio como un término adicional en el modelo de la brecha monetaria. Un modelo de brecha de dinero teóricamente congruente debería ser capaz de explicar variaciones en los precios de los bienes comerciables y no comerciables. Adicionalmente, el tipo de cambio se determina en gran medida por la evolución del dinero; la inclusión de ambas variables genera problemas de multicolinealidad.
} 


\section{Referencias}

Altimari, S. N. 2001. "Does Money Lead Inflation in the Euro Area?" European Central Bank Working Paper No. 63.

Armour, J., J. Atta-Mensah, W. Engert, y S. Hendry. 1996. "A Distant Early Warning Model of Inflation Based on M1 Disequilibria." Bank of Canada Working Paper No. 96-05.

Blanchard, O. J. y D. Quah. 1989. "The Dynamic Effect of Aggregate Demand and Supply Disturbances." American Economic Review 79: 655-73.

Blinder, A. S. 1998. Central Banking in Theory and Practice. Cambridge, Mass.: MIT Press.

Callen, T. y Chang, D. 1999. "Modelling and Forecasting Inflation in India." IMF Working Paper WP/99/119.

Campbell, J.Y. y P. Perron. 1991. "Pitfalls and Opportunities: What Macroeconomists Should Know about Unit Roots." En NBER Macroeconomics Annual 1991, ed. por S. Fischer. Cambridge, Mass.: MIT Press.

Catão, L. y M. Terrones. 2001. "Fiscal Deficits and Inflation: A New Look at the Emerging Market Evidence." IMF Working Paper WP/01/74.

Coe. D. T. y C. J. McDermott. 1999. "Does the Gap Model Work in Asia?" IMF Staff Papers 44(1): 59-80.

De Brower, G. y N. Ericsson. 1998. "Modelling Inflation in Australia." Journal of Business and Economic Statistics vol. 16, no. 4.

DeSerres, A., A. Guay y P. St-Amant. 1995. "Estimating and Projecting Potential Output Using Structural VAR Methodology." Bank of Canada Working Paper no. 95-2.

Duguay, P. 1994. "Empirical Evidence on the Strength of the Monetary Transmission Mechanism in Canada: An Aggregate Approach." Journal of Monetary Economics 33: 39-61.

Engert, W. y J. Selody. 1998. "Forecasting Inflation with the M1-VECM: Part Two." Bank of Canada Working Paper 98-6.

Engert, W. y J. Selody. 1998. "Uncertainty and Multiple Paradigm of the Monetary Transmission Mechanism." Bank of Canada Working Paper 98-7.

Fillion, J.-F. y A. Léonard. 1997. "La courbe de Phillips au Canada: un examen de quelques hypothèses." Bank of Canada Working Paper 97-3. 
Fischer, S., R. Sahay y C. Vegh. 2002. "Modern Hyper- and High Inflations" Journal of Economic Literature, forthcoming.

Fischer, S. 1994. "Modern Central Banking." In The Future of Central Banking, ed. por F. Capie, C. Goodhart, S. Fischer y N. Schnadt. New York: Cambridge University Press.

Freedman, C. 2000. "Monetary Aggregates and Monetary Policy in the Twenty-First Century: Discussion." In The Evolution of Monetary Policy and the Federal Reserve System Over the Past Thirty Years: A Conference in Honour of Frank Morris, Conference Series No. 45, Federal Reserve Bank of Boston.

Friedman, M. 1968. "The Role of Monetary Policy." American Economic Review 58: 1-17.

Fung, B.S.C. y M. Kasumovich. 1998. "Monetary Shocks in the G-6 Countries: Is There a Puzzle?" Journal of Monetary Economics 42(3): 575-92.

Gali, J., M. Gertler y J.D. López-Salido. 2001. "European Inflation Dynamics." European Economic Review 45(7): 1237-70.

Gamboa, R. y F. Aportela. 2001. "La Política Fiscal y la Inflación: el Caso de México". Banco de México, mimeo, forthcoming in "La Inflación en México", Gaceta de Economía, ITAM.

Garcés Díaz, D. G. 1999. "Determinación del Nivel de Precios y la Dinámica Inflacionaria en México." Banco de México Documento de Investigación No. 9907.

Garcés Díaz, D. G. 2001. "Cambios de Régimen en el Pass-Through y la Incertidumbre Inflacionaria." Banco de México, mimeo.

Garcés Díaz, D. G. 2002. "Agregados Monetarios, Inflación y Actividad Económica en México.” Banco de México, Documento de Investigación No. 2002-17.

García, C. y J. Restrepo. 2001. "Price Inflation and Exchange Rate Passthrough in Chile." Banco de Chile Working Paper no. 128.

González, J. 1998. "Regímenes Cambiarios y el Traspaso del Tipo de Cambio: ¿Hay un Enigma Mexicano?" El Trimestre Económico, 65(1): 49-97.

Goodfriend, M. y R. King. 1997. "The New Neoclassical Synthesis." In NBER Macroeconomics Annual 1997, ed. by B. Bernanke and J. Rotemberg. Cambridge, Mass.: MIT Press.

Goodfriend, M. 1997. “A Framework for the Analysis of Moderate Inflations.” Journal of Monetary Economics 39(1): 45-66.

Gordon, R.J. 1997. "The Time-Varying NAIRU and its Implications for Economic Policy." Journal of Economic Perspectives 11(1): 11-32. 
Graff, J. 1995. "Comportamiento de los Precios de Exportación de Productos Manufacturados: México, 1980-1994." Banco de México, Documento de Investigación No. 9505.

Hallman, J.J., Porter, R.D. y Small, D. H. 1991. "Is the Price Level Tied to the M2 Monetary Aggregate in The Long Run?" American Economic Review 81(4): 841-858.

Hansen, B. 1992. “Tests for Parameter Instability in Regressions with I(1) Processes.” Journal of Economic and Business Statistics 10: 321-335.

Hendry, S. 1995. "Long-Run Demand for M1." Bank of Canada Working Paper 95-11.

International Monetary Fund. 1996. "The Rise and Fall of Inflation: Lessons from the Postwar Experience." World Economic Outlook, October, Chapter VI. Washington, D.C.: International Monetary Fund.

2001. "The Decline of Inflation in Emerging Markets: Can It Be Maintained?" World Economic Outlook, May, Chapter IV. Washington, D.C.: International Monetary Fund.

Jácome H., L. 2001. "Legal Central Bank Independence and Inflation in Latin America During the 1990s," IMF Working Paper WP/01/212.

Jonsson, G. 1999. "Inflation, Money Demand, and Purchasing Power Parity in South Africa." IMF Working Paper WP/99/122.

Kenny, G. y D. McGettigan. 1999. "Modelling Traded, Non-Traded and Aggregate Inflation in a Small Open Economy: the Case of Ireland.” The Manchester School 67(1): 60-88.

Kichian, M. 2001. "On the Nature and Stability of the Canadian Phillips Curve." Bank of Canada Working Paper 2001-4.

King, M. 2002. "No money, no inflation—the role of money in the economy." Bank of England Quarterly Bulletin, summer 2002.

Longworth, D. y C. Freedman. 2000. "Models, Projections and the Conduct of Policy at the Bank of Canada." Paper prepared for the conference "Stabilization and Monetary Policy: The International Experience," Banco de México, November 14-15.

Lougani, P. y P. Swagel. 2001. "Sources of Inflation in Developing Countries." IMF Working Paper WP/01/198.

Macklem, T. 1997. "Capacity Constraints, Price Adjustment and Monetary Policy." Bank of Canada Review, Spring.

Messmacher, M. 2000. "Stabilisation Policies in Mexico: 1980-2000". Banco de México. Mimeo.

Messmacher, M. and A. Werner. 2001 "La Inflación en México:1950-2000." In La Inflación en 
México, Gaceta de Economía, ITAM, forthcoming.

Mohanty, M. S. y M. Klau. 2001. "What determines inflation in emerging market countries?" BIS Papers No. 8: Modelling aspects of the inflation process and the monetary transmission mechanism in emerging market countries.

Pérez-López, A. 1996 "Un Estudio Econométrico sobre la Inflación en México." Banco de México Research Paper no. 9604.

Phillips, A.W. 1958. "The Relationship Between Unemployment and the Rate of Change of Money Wages in the United Kingdom, 1861-1957." Económica 25: 283-299.

Phillips, A. y B. Hansen. 1990. "Statistical Inference in Instrumental Variable Regression with I(1) Processes.” Review of Economic Studies 57: 99-125.

Santaella, J. A. 2001. "El Traspaso inflacionario del Tipo de Cambio, la Paridad del Poder de Compra y Anexas: La Experiencia Mexicana." en La Inflación en México, Gaceta de Economía, ITAM, Tomo I, pp. 427-464.

Schwert, G.W. 1989. "Tests for Unit Roots: A Monte Carlo Investigation." Journal of Business and Economic Statistics 7: 147-159.

Simone, F. 2000. "Forecasting Inflation in Chile Using State-Space and Regime-Switching Models." IMF Working Paper WP/ 00/162.

Springer, P. y M. Kfoury. 2002. “A Simple Model for Inflation Targeting in Brazil.” Brazilian Journal of Applied Economics 6(1): 31-48.

Stock, J.H., y M.W. Watson. 1999. "Forecasting Inflation." Journal of Monetary Economics 44: 293-335.

Torres, A. 2000. "Estabilidad en Variables Nominales y el Ciclo Económico: El Caso de México" Banco de México Research Paper no. 2000-3.

Werner, A. y J. Ardavín. 2001 "Objetivos de Inflación y Finanzas Públicas." In La Inflación en México, Gaceta de Economía, ITAM, Tomo II, pp. 207-230. 


\section{Apéndice 1: Gráficas}

\section{Gráfica 2}

Imflación del INPC Trimestre a Trimestre 1983:01 a 2001:04

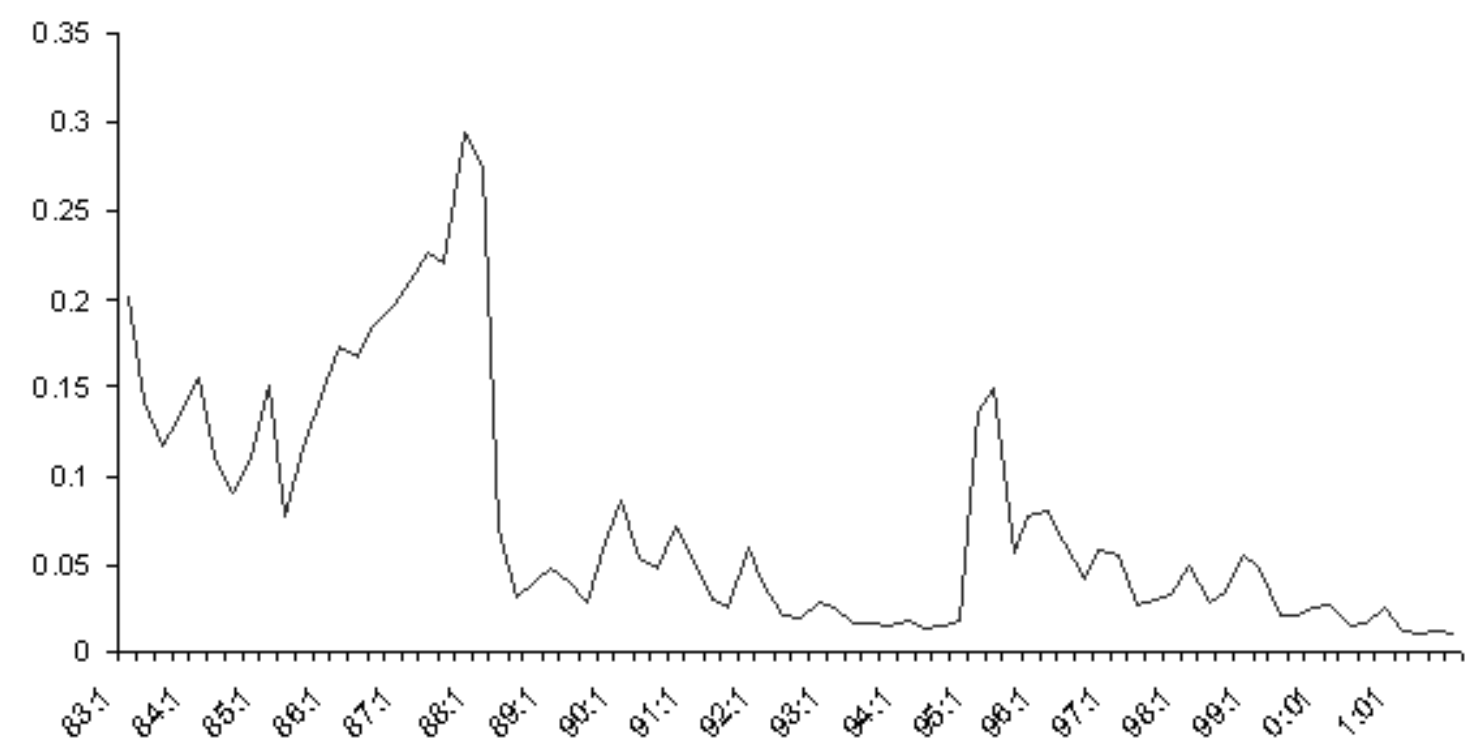

\section{Gráfica 3}

Tasa de Depreciación del Tipo de Cambio Peso/Dólar 1983:01 a 2001:04

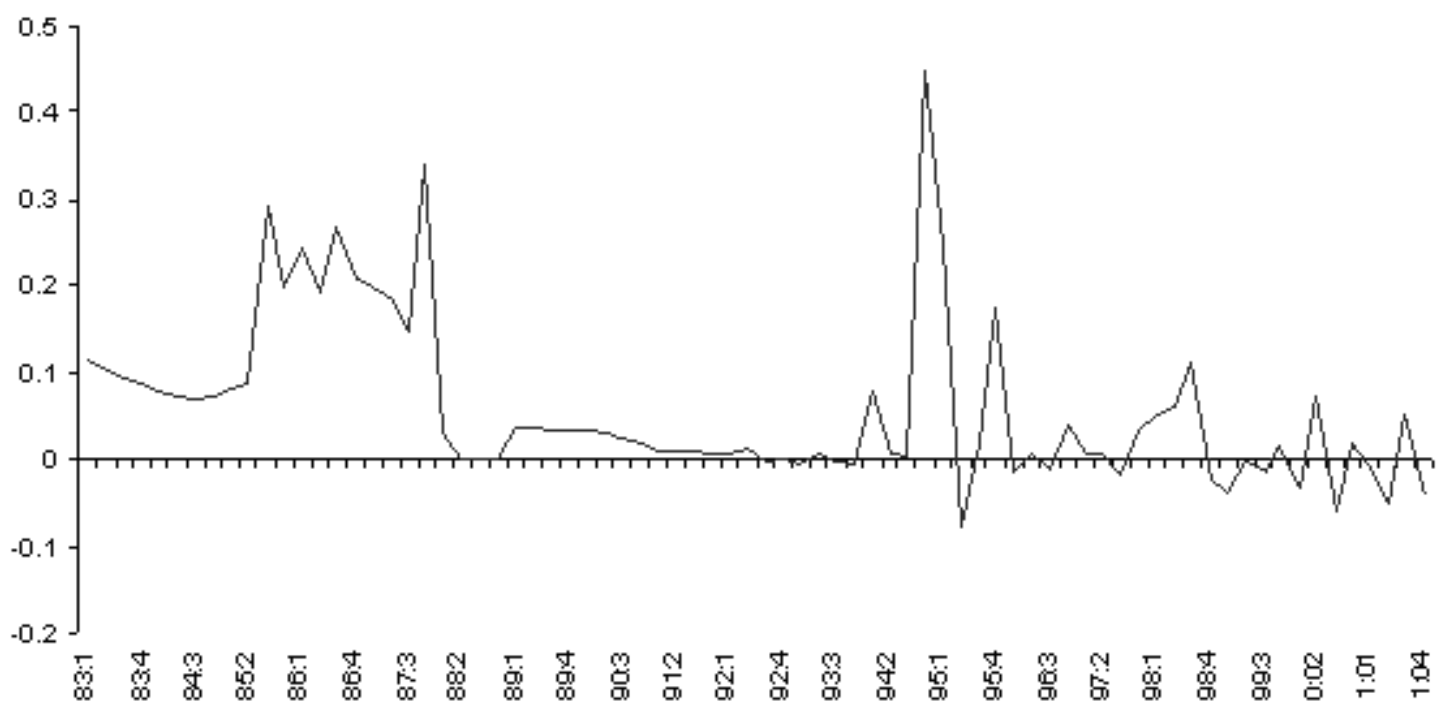




\section{Gráfica 4}

\section{Brecha de Producto en México Estimada Usando un SVAR de 3 Variables 1983:01-2001:04}

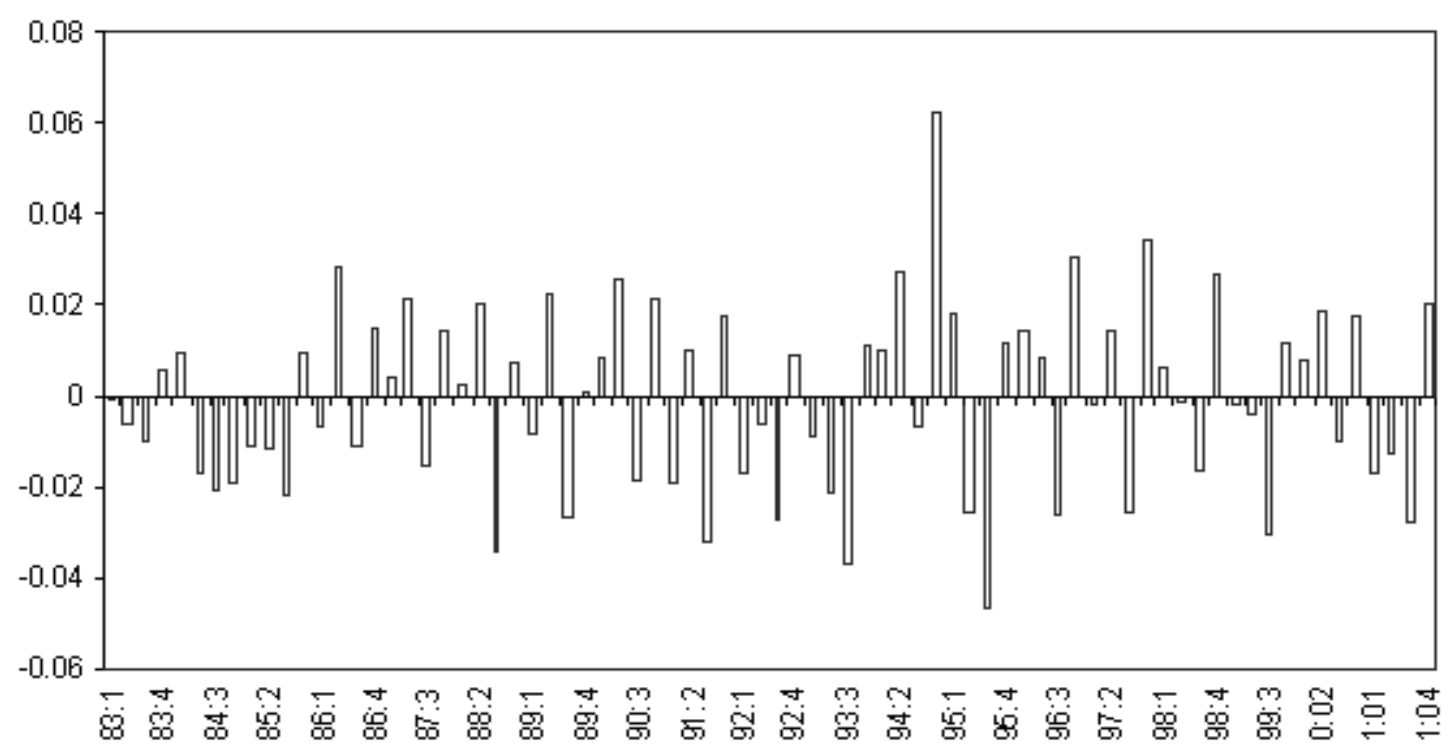

\section{Gráfica 5}

PIB Observado y PIB Potencial Estimado con un SVAR de 3 Variables 1983:1-2001:04

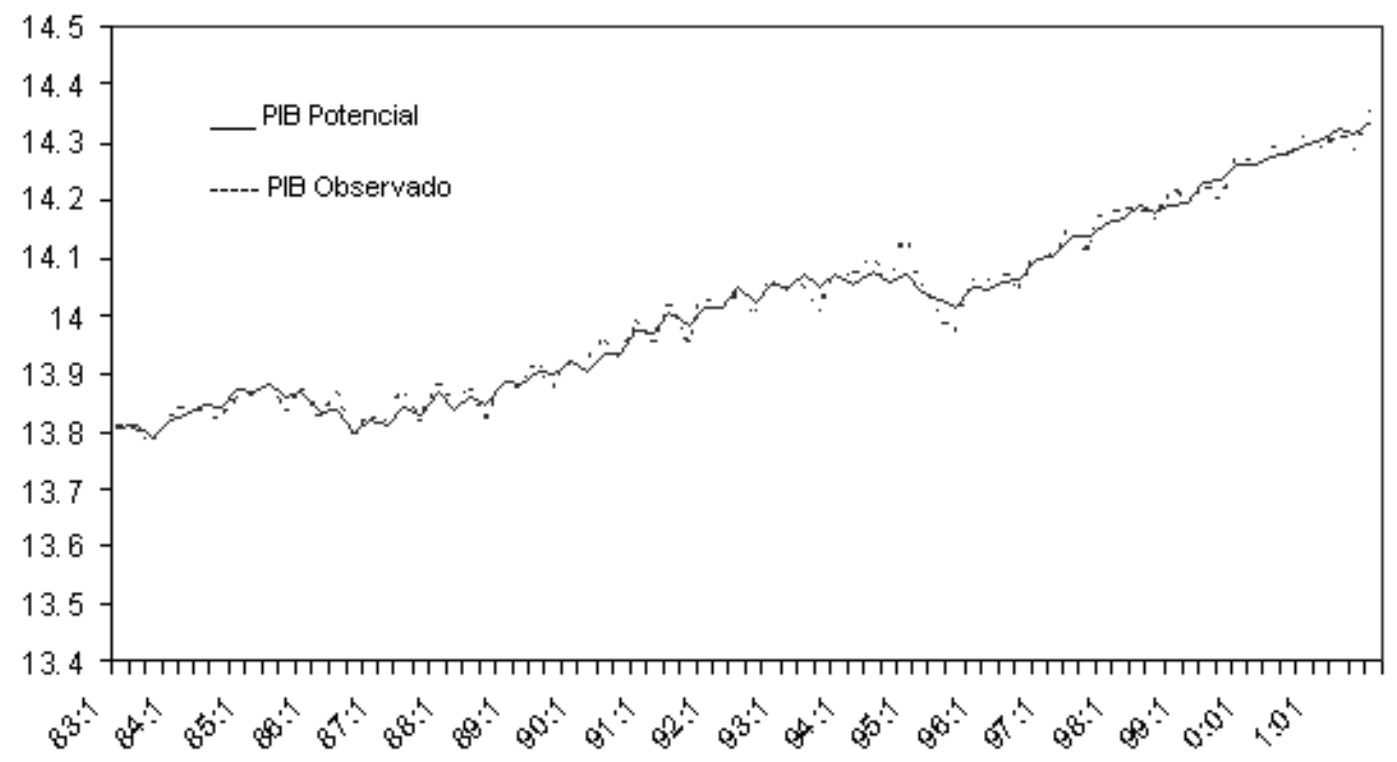




\section{Apéndice 2: Resultados de la Estimación y Pronósticos}

Cuadro 2.1: Resultados de la Estimación 1983:1-2001:4 Variable Dependiente: $\Delta \mathbf{p}_{\mathbf{t}}(\mathbf{t} / \mathbf{t})$

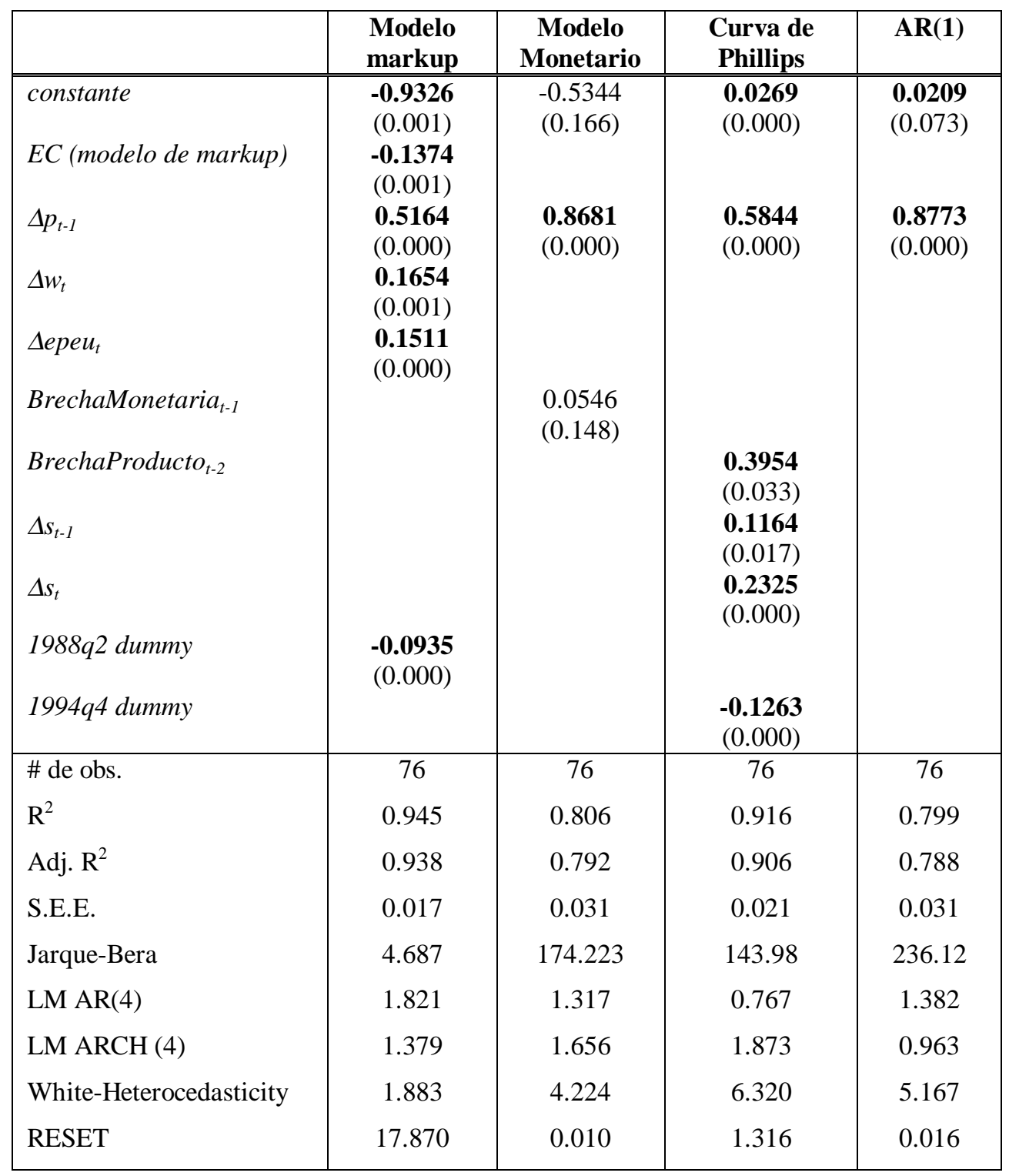

Notas: Los números entre paréntesis son los valores p. Valores en negritas indican significación al menos al nivel del $10 \%$. En cada regresión se incluyeron variables estacionales. Los errores estándar consideran posibles problemas de heterocedasticidad. 


\section{Apéndice 2: Resultados de Estimación y Pronóstico}

\section{Cuadro 2.2 Desempeño del Pronóstico Fuera de Muestra (Dinámico) \\ Usando los Valores Observados de las Variables Explicativas \\ Período de Estimación: 1983Q1-1996Q4 \\ Período de Pronóstico: 1997Q1-2001Q4}

\begin{tabular}{|l|c|c|c|c|}
\hline & $\begin{array}{c}\text { Modelo } \\
\text { markup }\end{array}$ & $\begin{array}{c}\text { Modelo } \\
\text { Monetario }\end{array}$ & $\begin{array}{c}\text { Curva de } \\
\text { Phillips }\end{array}$ & AR(1) \\
\hline \hline Raíz del Error Cuadrático Medio (RMSE) & 0.037 & 0.094 & 0.061 & 0.084 \\
Error Medio Absoluto (MAE) & 0.031 & 0.074 & 0.048 & 0.075 \\
Error Medio (ME) & -0.031 & 0.063 & 0.042 & 0.075 \\
\hline
\end{tabular}

\section{Cuadro 2.3 Desempeño del Pronóstico Fuera de Muestra (Dinámico) Usando los Valores Pronosticados de las Variables Explicativas Período de Estimación: 1983Q1-1996Q4 Período de Pronóstico: 1997Q1-2001Q4}

\begin{tabular}{|l|c|c|c|c|}
\hline & $\begin{array}{c}\text { Modelo } \\
\text { markup }\end{array}$ & $\begin{array}{c}\text { Modelo } \\
\text { Monetario }\end{array}$ & $\begin{array}{c}\text { Curva de } \\
\text { Phillips }\end{array}$ & AR(1) \\
\hline \hline Raíz del Error Cuadrático Medio (RMSE) & 0.038 & 0.105 & 0.155 & 0.084 \\
Error Medio Absoluto (MAE) & 0.033 & 0.085 & 0.133 & 0.075 \\
Error Medio (ME) & -0.033 & 0.069 & 0.123 & 0.075 \\
\hline
\end{tabular}

\section{Apéndice 3: Resultados de las Pruebas de Raíz Unitaria y Cointegración}

Cuadro 3.1: Resultados de las Pruebas de Raíz Unitaria en las Variables Usadas en la Curva de Phillips 1983:1-2001:4

\begin{tabular}{|l|c|c|c|c|}
\hline \multicolumn{1}{|c|}{ Series } & $\begin{array}{c}\text { ADF } \\
\text { (sin tend.) }\end{array}$ & $\begin{array}{c}\text { ADF } \\
\text { (con tend.) }\end{array}$ & $\begin{array}{c}\text { PP } \\
\text { (sin tend.) }\end{array}$ & $\begin{array}{c}\text { PP } \\
\text { (con tend.) }\end{array}$ \\
\hline Inflación del INPC & $-2.25(0.19)$ & $\mathbf{- 3 . 2 0}(0.08)$ & $\mathbf{- 1 4 . 6 0}(0.08)$ & $\mathbf{- 2 3 . 7 0}(0.04)$ \\
\hline Tipo de Cambio & $-2.52(0.11)$ & $-2.44(0.36)$ & $-2.38(0.27)$ & $-2.93(0.06)$ \\
\hline Tipo de Cambio (d) & $-2.13(0.23)$ & $-2.79(0.20)$ & $\mathbf{- 2 8 . 7 2}(0.01)$ & $\mathbf{- 3 8 . 0 0}(0.00)$ \\
\hline Brecha Producto (\%) & $\mathbf{- 4 . 9 1 ( 0 . 0 0 )}$ & $\mathbf{- 5 . 1 6}(0.00)$ & $\mathbf{- 6 5 . 0 3}(0.00)$ & $\mathbf{- 6 8 . 4 8}(0.00)$ \\
\hline
\end{tabular}

Notas: Las columnas etiquetadas con "ADF" y "PP" reportan los estadísticos de prueba de Dickey-Fuller aumentada y pruebas Phillips-Perron, respectivamente. Los números entre paréntesis son los valores p. Los números en negritas indican significación al menos al 10\%. 
Cuadro 3.2: Resultados de las Pruebas de Raíz Unitaria de las Variables en el VAR Estructural 1980:1-2001:4

\begin{tabular}{|l|c|c|c|c|}
\hline \multicolumn{1}{|c|}{$\begin{array}{c}\text { Series } \\
\text { (en Logs) }\end{array}$} & $\begin{array}{c}\text { ADF } \\
\text { (sin tend.) }\end{array}$ & $\begin{array}{c}\text { ADF } \\
\text { (con tend.) }\end{array}$ & $\begin{array}{c}\text { PP } \\
\text { (sin tend.) }\end{array}$ & $\begin{array}{c}\text { PP } \\
\text { (con tend.) }\end{array}$ \\
\hline Precio Real Petróleo (nivel) & $-1.98(0.29)$ & $-2.54(0.31)$ & $-4.76(0.46)$ & $-10.20(0.39)$ \\
\hline Precio Real Petróleo (diferencia) & $\mathbf{- 5 . 0 8}(0.00)$ & $\mathbf{- 5 . 2 8}(0.00)$ & $\mathbf{- 5 5 . 5 1}(0.00)$ & $\mathbf{- 5 4 . 4 4 ( 0 . 0 0 )}$ \\
\hline PIB Real (nivel) & $\mathbf{1 . 6 5}(0.00)$ & $-1.56(0.19)$ & $\mathbf{- 0 . 0 8}(0.05)$ & $\mathbf{- 4 0 . 0 8 ( 0 . 0 0 )}$ \\
\hline PIB Real (diferencia) & $\mathbf{- 3 . 0 5 ( 0 . 0 3 )}$ & $\mathbf{- 3 . 8 9}(0.01)$ & $\mathbf{- 1 4 1 . 1}(0.00)$ & $\mathbf{- 1 3 9 . 2 3}(0.00)$ \\
\hline Tipo de Cambio Real (nivel) & $\mathbf{- 3 . 3 0}(0.01)$ & $\mathbf{- 3 . 7 2}(0.02)$ & $-11.36(0.14)$ & $-11.26(0.36)$ \\
\hline Tipo de Cambio Real (diferencia) & $\mathbf{- 3 . 6 8 ( 0 . 0 0 )}$ & $\mathbf{- 3 . 8 7}(0.01)$ & $\mathbf{- 1 0 0 . 5}(0.00)$ & $\mathbf{- 9 8 . 4 8}(0.00)$ \\
\hline
\end{tabular}

Notas: Las columnas etiquetadas con "ADF" y "PP" reportan los estadísticos de prueba de Dickey-Fuller aumentada y Phillips-Perron tests, respectivamente. Los números entre paréntesis son los valores p. Los números en negritas indican significatividad al menos al $10 \%$.

\section{Cuadro 3.3: Resultados de las Pruebas de Cointegración de las Variables en el VAR Estructural 1980:1-2001:4}

\begin{tabular}{|l|c|c|}
\hline & $\lambda_{\max }$ & $\lambda_{\text {trace }}$ \\
\hline $\mathrm{H}_{0}: \mathrm{r}=0$ & 15.84 & 22.23 \\
\hline Critical value (95\%) & 20.78 & 29.51 \\
\hline
\end{tabular}

Notas: Las columnas con las etiquetas " $\lambda_{\max }$ " y " $\lambda_{\text {trace }}$ " reportan los estadísticos de prueba y los valores críticos usados en la prueba de cointegración de Johansen. En ambos casos, la hipótesis nula es la ausencia de cointegración. El número de rezagos fue seleccionado con la estrategia general a específico.

\section{Apéndice 4: Estimación del Producto Potencial Utilizando la Metodología de un VAR Estructural: Ecuaciones Clave ${ }^{35}$}

Supóngase que el precio internacional del petróleo (oil), el PIB real mexicano (y) y el tipo de cambio real bilateral México/Estados Unidos (rer) siguen un proceso estacionario en forma de primeras diferencias que responden a tres tipos de perturbaciones ortogonales: perturbaciones al precio del petróleo $\left(\varepsilon_{\mathrm{oil}}\right)$, otras perturbaciones de oferta $\left(\varepsilon_{\mathrm{s}}\right)$ y perturbaciones de demanda $\left(\varepsilon_{\mathrm{d}}\right)$. Así, estas variables se determinan conjuntamente en este sistema. El modelo estructural correspondiente a este sistema puede tener la siguiente representación de promedios móviles:

$$
\Delta x_{t}=A_{0} \varepsilon_{t}+A_{1} \varepsilon_{t-1}+\ldots=\sum_{i=0}^{\infty} A_{i} \varepsilon_{t-i}=A(L) \varepsilon_{t}
$$

\footnotetext{
${ }^{35}$ Esta sección se basa en Deserres, Guay y St-Amant (1995)
} 
donde

$$
\varepsilon_{t}=\left[\begin{array}{c}
\varepsilon_{o i l} \\
\varepsilon_{s} \\
\varepsilon_{d}
\end{array}\right] \text { and } \Delta x_{t}=\left[\begin{array}{c}
\Delta o i l \\
\Delta y \\
\Delta r e r
\end{array}\right]
$$

y la varianza de las perturbaciones estructurales se normalizan de tal modo que $E\left(\varepsilon_{\mathrm{t}} \varepsilon_{\mathrm{t}}\right)=\mathrm{I}$.

Para poder identificar este modelo estructural, la forma reducida del modelo VAR dada por:

$$
\Delta x_{r}=B_{1} \Delta x_{t-1}+\ldots+B_{q} \Delta x_{t-q}+e_{t}
$$

debe ser estimada primero, donde $e_{t}$ es el vector de residuales estimados, q es el número de rezagos, y $\mathrm{E}\left(\mathrm{e}_{\mathrm{t}} \mathrm{e}_{\mathrm{t}}\right)=\Sigma$. Dado que el proceso estocástico es estacionario, (9) puede ser re-escrita como un proceso de promedios móviles de orden infinito como sigue:

$$
\Delta x_{t}=e_{t}+C_{1} e_{t-1}+\ldots=\sum_{i=0}^{\infty} C_{i} e_{t-i}=C(L) e_{t}
$$

Esta representación del proceso es única y puede ser obtenida estimando primero e invirtiendo después la representación VAR de la ecuación (9). Así, los residuales de la forma reducida del modelo están relacionados con los residuales estructurales como sigue:

$$
\varepsilon_{t}=A_{0}^{\prime} e_{t}
$$

lo cual implica que:

$$
E\left(e_{t} e_{t}\right)=A_{0} E\left(\varepsilon_{t} \varepsilon_{t}\right) A_{0}^{\prime}
$$

y así,

$$
A_{0} A_{0}^{\prime}=\Sigma
$$

Para poder estimar las perturbaciones estructurales $\varepsilon$ a partir de las perturbaciones de la forma reducida $e$ (encontrada al estimar la ecuación (9)), es necesario imponer algunas restricciones de identificación para evaluar los elementos de $\mathrm{A}_{0}$. Dado que se tiene un sistema de tres variables, $\mathrm{A}_{0}$ tiene nueve elementos. Puesto que la matriz de varianza-covarianza estimada $\Sigma$ es simétrica, la ecuación (13) provee seis restricciones de identificación independientes. Por tanto, es necesario imponer tres restricciones adicionales provenientes de (7), (11) y (13). Se aprecia que la matriz de efectos de largo plazo de las perturbaciones de la forma reducida, $\mathrm{C}(1)$, está relacionada a la matriz equivalente de las perturbaciones estructurales, A(1), de la siguiente manera:

$$
A(1)=C(1) A_{0}
$$

donde la matriz $\mathrm{C}(1)$ se calcula a partir del VAR estimado. Por ello, se imponen tres restricciones de identificación sobre A(1), basadas la teoría económica. La primera sigue del supuesto de que las perturbaciones de demanda no tienen efectos permanentes sobre el producto. Siguiendo a Blanchard y Quah (1989), entre otros, esto implica que todas las perturbaciones de demanda tienen 
un efecto transitorio sobre el producto son interpretados como perturbaciones de demanda. La segunda y tercera restricciones, las cuales permiten distinguir entre perturbaciones del precio del petróleo y otras perturbaciones de oferta, están basadas en el supuesto de que solamente las primeras tienen efectos de largo plazo sobre el precio del petróleo.

Se obtiene la siguiente descomposición estructural para el producto:

$$
\Delta y_{t}=\mu+A_{o i l}(L) \varepsilon_{\text {oilt }}+A_{s}(L) \varepsilon_{s t}+A_{d}(L) \varepsilon_{d t}
$$

donde los movimientos en el producto pueden ser descompuestos en combinaciones de promedios móviles de los diferentes tipos de perturbaciones más la tendencia determinística en el producto $(\mu)$. Los tres primeros términos a la derecha de la ecuación (15) representan la medición del producto potencial en forma de primera diferencia. 